\title{
On linear waveguides of square and triangular lattice strips: an application of Chebyshev polynomials
}

\author{
BASANT LAL SHARMA \\ Department of Mechanical Engineering, Indian Institute of Technology Kanpur, Kanpur 208016, India \\ e-mail: bls@iitk.ac.in
}

MS received 18 February 2016; revised 13 October 2016; accepted 30 November 2016

\begin{abstract}
An analysis of the linear waves in infinitely-long square and triangular lattice strips of identical particles with nearest neighbour interactions for all combinations of fixed and free boundary conditions, as well as the periodic boundary, is presented. Expressions for the dispersion relations and the associated normal modes in these waveguides are provided in the paper; some of which are expressed implicitly in terms of certain linear combinations of the Chebyshev polynomials. The effect of next-nearest-neighbour interaction is also included for the square lattice waveguides. It is found that localized propagating waves, so called surface wave modes, occur in the triangular lattice strips, as well as square lattice strips with next-nearest-neighbour interactions, when either or both boundaries are free. In this paper, the even and odd modes are also discussed separately, wherever applicable. Graphical illustrations of the dispersion curves are included for all waveguides. The discrete waveguides analysed in the paper have broad applications in physics and engineering, including their merit in classical problems in elasticity, acoustics and electromagnetism, as well as recent technological issues involving various transport phenomena in quasi-one-dimensional nano-structures.
\end{abstract}

Keywords. Dispersion; normal modes; square; triangular; Chebyshev; tridiagonal.

\section{Introduction}

In classical elastic waveguides $[1,2]$ such as plates, rods, cylindrical shells, layered solids, etc., the 'neighbouring' parallel boundaries 'guide' the waves [3-5]. In fact, the research works on elastic waveguides can be traced to 1876 $[6,7]$, slightly before the well-known contributions due to Rayleigh [8,9] and Lamb [10, 11]. Pochhammer transformed the three-dimensional equation of motion of a solid circular rod into cylindrical coordinates, and for (traction-) free surfaces, derived the dispersion relation [12] for each mode (see also the related works of [13-17]). The technological innovations and literature reviews involving the electromagnetic counterparts, which had a greater impact in communication technology, are historically more numerous $[18,19]$ (see also, for example, [20, 21], and [22, Chapter 10]). These analyses play a pivotal role in, the so called, mode-matching techniques for wave propagation in structured waveguides such as bifurcated waveguides, waveguides with discontinuities, etc. [23-27]. Indeed, any arbitrary time periodic motion of a given waveguide can be expressed in terms of the normal modes. In contrast with the continuous waveguides, the discrete waveguides allow an interpretation in terms of infinitely long mutually coupled chains of particles; this leads to a quasi-one-dimensional point of view. The wave propagation analysis for continuous and discrete waveguides also finds many physical applications, such as pure crystals dynamics (simple, complex, etc.) in harmonic approximation [12, 28, 29] and also in the presence of impurities [30, 31], vibrations of macromolecules appearing in biological [32], chemical applications [33], etc. The technological interests, some of them quite recent, involving phononic [34, 35], photonic [35-37], electronic [38-41], phonon [35, 37, 42-45], mechanical [46-48] and magnetic-spin related transport [49-51], in nanostructures cannot be excluded as well; these apparently diverse applications involve the governing equations, which have similar mathematical structure, sometimes identical, to those analysed in this document.

The paper presents an analysis of simplified models of lattice waveguides with two types of structure: square and triangular, assuming nearest-neighbour interactions and identical nature of particles [52-54], from the point of view of linear propagating waves. A schematic illustration of the discrete structure in 'classical' waveguides is shown in figure 1. Both types of lattice models also occur in the research works on brittle fracture (see [55], and a large number of references provided in its bibliography), although the explicit nature of wave propagation, in square and triangular lattice waveguides, has not been analysed, so far, as part of these problems in dynamic fracture. Additionally, the present paper places the results, peculiar to 


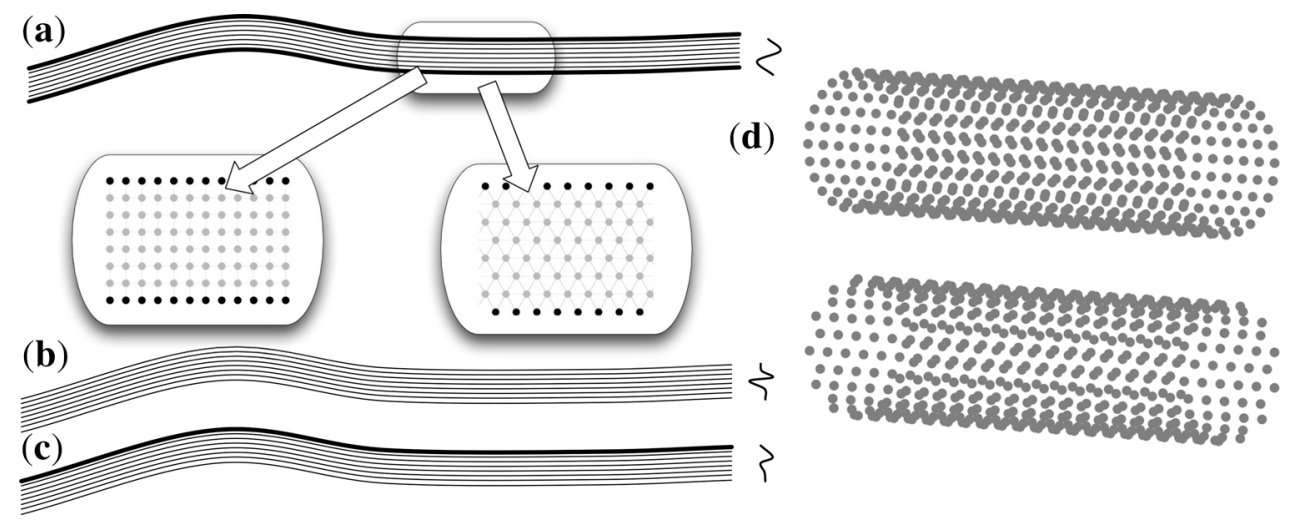

Figure 1. Illustration of lattice waveguide structure with upper-lower boundary: (a) fixed-fixed, (b) free-free and (c) fixed-free, along with a small snapshot of the mode shape across the waveguide 'cross-section' and (d) a 'tube' of square and triangular lattice waveguides undergoing a 'small' radial motion.

linear wave propagation, using the Chebyshev polynomials [56-58]. The latter are ubiquitous in numerical analysis, but they are also known to find applications in wave mechanics [59]. Several identities are available for the Chebyshev polynomials, owing to their historical importance and huge number of applications in science [58], some of which find applications in this paper. Due to a quasi-one-dimensional nature of the lattice waveguides, it can be anticipated that an important role is played by the structured matrices (tridiagonal, circulant, Toeplitz matrices, etc.) [60]. The paper presents an application of the analytical expression of the eigenvalues and eigenvectors of tridiagonal matrices that have two or four of their corners perturbed [61] (also see $[60,62,63])$. As an alternative method, which is in fact needed for triangular lattice waveguide with free boundary conditions, the manipulations elaborated in [55] and utilized in [64, 65] (and [66]) also find an application in the same context. The paper considers all combinations of fixed and free boundary conditions for the lattice waveguides, in addition to the periodic boundary condition (see figure 1). For each case of identical upper and lower boundaries, the even and odd modes are also discussed. The dispersion relations and normal modes for the propagating waves are derived in detail for each waveguide. The effect of a larger number of interactions is also discussed but to a limited extent. The square lattice waveguide is chosen for an illustration of the influence of the nearest-neighbour interactions. Incidentally, the analysis carries over rather easily as an analogue of that for the triangular lattice. Indeed, higher order of neighbour interactions in the lattice models leads to several difficulties from the analytical point of view $[12,54]$. Besides this, the Chebyshev polynomials are anticipated to lose their relevance in this generalization due to the presence of banded matrices (with more than three 'diagonals', in place of the tridiagonal matrices, which are relatively well understood $[60,63,67-69])$; the corresponding study is relegated to another forum.

As one of the main results in this paper, it is found that the triangular lattice waveguides, as well as square lattice strips with next-nearest-neighbour interactions, allow the presence of localized modes, also called surface modes [70-73]. The identification of the actual locations of surface wave modes has potential utility in several fields [74-76], as well as myriad physical applications in parallel, as mentioned earlier. It is found that surface (localized) wave modes exist for the triangular lattice ${ }^{1}$ (even with the simplest form of interactions studied in this paper), as well as square lattice strips with next-nearest-neighbour interactions, whenever at least one of the boundary rows is free. Such surface wave modes do not exist for the simplified model of square lattice with only nearest-neighbour interactions, which is well known and expected due to its separable nature and similarity with the one dimensional lattice model having only nearest-neighbour interactions $[12,54]$. The reader may note that all dispersion relations and normal modes listed in this paper have been verified against a numerical solution of the discrete Helmholtz equation, in context, with given lattice waveguide boundary conditions.

\subsection{Notation}

Let $\mathbb{Z}$ denote the set of integers, $\mathbb{Z}^{2}$ denote $\mathbb{Z} \times \mathbb{Z}$. Let $\mathbb{Z}_{m}^{n}$ denote the set of integers $\{m, m+1, \ldots, n-1, n\}$. Let $\mathbb{R}$ denote the set of real numbers and $\mathbb{C}$ denote the set of complex numbers. The symbol $\xi$ is exclusively used throughout as a real variable that denotes a real wave number in the waveguide (horizontal) direction. Similarly, $\eta$ denotes the equivalent wave number along the vertical direction, which may be real or complex depending on the

\footnotetext{
1 The triangular lattice, according to the usage in this paper, is also known as hexagonal lattice in certain fields in engineering and sciences. Both lattice structures also appear in finite-difference-based numerical analysis [77], from the point of view of discretization of the traditional Laplacian.
} 
context. Latin letters $C_{1}, C_{2}$, etc., denote arbitrary constants in expressions. The notation for relevant physical entities is described in the main text.

\section{Mechanical lattice model}

Let $\left\{\mathbf{e}_{1}, \mathbf{e}_{2}\right\}$ be two orthonormal unit vectors in the twodimensional Euclidean space $\mathbb{R}^{2}$. Suppose $\rho$ is the threedimensional mass density of an elastic material, $\mu$ is the linear elastic shear modulus and $c_{s}$ is the macroscopic shear wave speed $\sqrt{\mu / \rho}$ [78]. Let a finite strip $S_{N}$ in $\mathbb{R}^{2}$ be constructed of such elastic material. A lattice strip is defined as a subset of the infinite lattice of particles embedded in $S_{N}$. Assume there are $N$ number of rows each containing infinite number of particles, arranged in the form of one-dimensional lattices. Both types of the lattice models, namely, square and triangular, contain identical particles, with each particle having mass $M$. In case of the first two models studied in this paper, it is assumed that only the in-plane nearest neighbours, separated by an equilibrium length $b$, are connected to each other by linearly elastic identical (massless) bonds to account for the relative displacement along anti-plane direction. The bonds have a spring constant $K$. In case of the third model, it is a square lattice with (linear) nextnearest-neighbour interactions having a spring constant $\gamma K$; other details of this model are provided later in the paper due to their resemblance with those for the first two, simpler, models. In addition to the natural interpretation of the lattice strips as two-dimensional layers undergoing out-of-plane motion, a three-dimensional interpretation of the same models is also possible; remarks of this order appear in $[64,65]$ for the first two models and are briefly included later as well.

\subsection{Square lattice waveguide $\mathfrak{\Xi}_{N}$}

Let $b_{1}=b_{2}=b$ and $\Im_{N}=\left\{\sum_{i=1,2} v_{i} b_{i} \mathbf{e}_{i} \mid v_{1} \in \mathbb{Z}, v_{2} \in \mathbb{Z}_{1}^{N}\right\}$ denote a two-dimensional square lattice strip. The antiplane displacement of a particle, indexed by its lattice coordinates $(x, y) \in \mathbb{Z}^{2}$, is denoted by $u_{x, y} \in \mathbb{C}$. Let $\mathbb{Z}_{N}^{2}$ denote the lattice coordinates of the particles in $\mathfrak{S}_{N}$, which have a degree of freedom to move in the anti-plane direction. The equation of motion of that portion of lattice waveguide $\mathfrak{\Im}_{N}$ which is away from the upper and lower boundary rows, while excluding the explicit dependence of $u$ on time $t$, is

$$
\begin{aligned}
M \frac{d^{2}}{d t^{2}} u_{x, y}= & K\left(u_{x+1, y}+u_{x-1, y}+u_{x, y+1}+u_{x, y-1}-4 u_{x, y}\right), \\
& (x, y) \in \mathbb{Z}_{N}^{2} .
\end{aligned}
$$

Motivated by a three-dimensional context, let $M=$ $\rho b^{3}, K=\mu b, t=t c_{s}$. In the context of $\Im_{N}$, this implies that
Table 1. Variants of $\subseteq_{N}$.

\begin{tabular}{lcc}
\hline S. no. & Strip & Remark \\
\hline 1 & $\mathfrak{S}_{0}^{:}$ & Even/odd modes \\
2 & $\mathfrak{S}_{0}^{\circ}$ & Even/odd modes \\
3 & $\mathfrak{S}_{\circ}^{:}$ & Asymmetric \\
4 & $\mathfrak{S}_{\odot}$ & - \\
\hline
\end{tabular}

the particles have a unit mass, the in-plane equilibrium spacing between the nearest neighbours is $b$ and the nearest-neighbour interactions (for the relative displacement in anti-plane direction) have spring constant $1 / b^{2}$. Evidently, the effect of boundary conditions on the force constants as well as lattice spacing is ignored in this simplified model. The square lattice dispersion relation $[52,64]$ is $\omega^{2}=$ $\frac{1}{b^{2}} \Omega_{\Im}^{2}(\xi, \eta)$, where

$$
\Omega_{\Im}^{2}(\xi, \eta)=4 \sin ^{2} \frac{1}{2} \xi+4 \sin ^{2} \frac{1}{2} \eta, \quad(\xi, \eta) \in[-\pi, \pi]^{2} .
$$

The representative variants of the square lattice waveguides along with boundary conditions are listed in table 1 (see also figure 2 and Remark 1). Using the lattice model formulation, the lattice waveguides of $\mathfrak{S}$ are decorated with the symbols $\bullet$ and $\circ$ as superscript (respectively subscript) to indicate the fixed and free upper (respectively lower) boundary condition, respectively, and $\odot$ to denote the periodic boundary condition.

\subsection{Triangular lattice waveguide $\mathfrak{I}_{N}$}

Let $b_{1}=\frac{1}{2} b, b_{2}=\frac{\sqrt{3}}{2} b$ and $\mathfrak{I}_{N}=\left\{\sum_{i=1,2} v_{i} b_{i} \mathbf{e}_{i} \mid v_{1} \in 2 \mathbb{Z}+\right.$ $\left.\bmod \left(\left|v_{2}\right|, 2\right), v_{2} \in \mathbb{Z}_{1}^{N}\right\}$ be a two-dimensional strip of a triangular lattice. Similar to the application by Sharma [65], a rectangular coordinate system is used here to allow certain convenience of labelling the slant arrangement of particles (alternative labels are used by Kantorovich and Krylov [77], Bilbao [79], Collatz [80] and others as examples). A replica of $\mathfrak{I}_{N}$, called a 'replicated' triangular (sub-)lattice strip and denoted by $\mathfrak{I}_{N}{ }^{\mathrm{R}}$, is juxtaposed with $\mathfrak{I}_{N}$, after imparting it a horizontal translation of magnitude $b_{1}$. The union of both lattice strips is a rectangular lattice strip, denoted by $\mathfrak{R}_{N}$, with a period $b_{1}$ horizontally and $b_{2}$ vertically. Let the displacement of a particle in $\mathfrak{R}_{N}$, indexed by its lattice coordinates $(x, y) \in \mathbb{Z}^{2}$, be denoted by $u_{x, y} \in \mathbb{C}$. Let $\mathbb{Z}_{N}^{2}$ denote the lattice coordinates of the particles in $\mathfrak{I}_{N}$, which have a degree of freedom to move in the antiplane direction. The equation of motion of the lattice, while suppressing the explicit dependence of $u$ on time $t$, is 


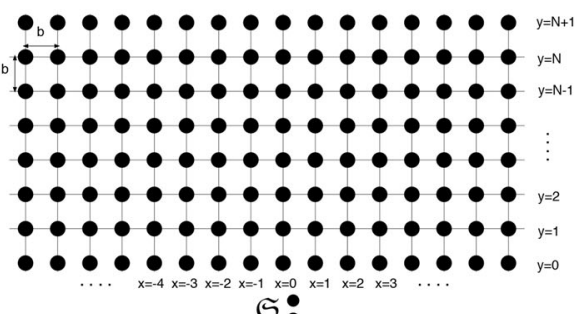
$5:$

$\bullet \bullet \bullet \bullet \bullet \bullet \bullet \bullet \bullet \bullet \bullet \bullet \bullet \bullet \bullet \mathrm{y}_{\mathrm{y}} \mathrm{N}$ $\bullet \bullet \bullet \bullet \bullet \bullet \bullet \bullet \bullet \bullet \bullet \bullet \bullet \bullet \bullet y^{y}=N-1$ $\bullet \bullet \bullet \bullet \bullet \bullet \bullet \bullet \bullet \bullet \bullet \bullet \bullet \bullet \bullet$ $\bullet \bullet \bullet \bullet \bullet \bullet \bullet \bullet \bullet \bullet \bullet \bullet \bullet \bullet \bullet \bullet$ $\bullet \bullet \bullet \bullet \bullet \bullet \bullet \bullet \bullet \bullet \bullet \bullet \bullet \bullet \bullet \mathrm{y}=$

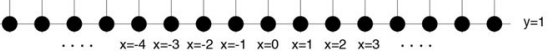
$\mathfrak{5} \circ$

$\bullet \bullet \bullet \bullet \bullet \bullet \bullet \bullet \bullet \bullet \bullet \bullet \bullet \bullet \bullet \mathrm{y} \mathrm{N}+1$ $\bullet \bullet \bullet \bullet \bullet \bullet \bullet \bullet \bullet \bullet \bullet \bullet y^{N} N$

$\bullet \bullet \bullet \bullet \bullet \bullet \bullet \bullet \bullet \bullet \bullet \bullet \bullet^{\mathrm{y}=\mathrm{N}-1}$ $\bullet \bullet \bullet \bullet \bullet \bullet \bullet \bullet \bullet \bullet \bullet \bullet \bullet \bullet$ $\bullet \bullet \bullet \bullet \bullet \bullet \bullet \bullet \bullet \bullet \bullet \bullet$ $\bullet \bullet \bullet \bullet \bullet \bullet \bullet \bullet \bullet \bullet \bullet \bullet \bullet \bullet \bullet \mathrm{y}=2$ $\bullet \bullet \bullet \bullet \bullet \bullet \bullet \bullet \bullet \bullet \bullet \bullet \bullet y_{y}=1$ $\mathfrak{S}:$

$\bullet \bullet \bullet \bullet \bullet \bullet \bullet \bullet \bullet \bullet \bullet \bullet \mathrm{y}=\mathrm{N}$ $\bullet \bullet \bullet \bullet \bullet \bullet \bullet \bullet \bullet \bullet y_{y=N}$

$\bullet \bullet \bullet \bullet \bullet \bullet \bullet \bullet \bullet \bullet \mathrm{y}^{\mathrm{N}-\mathrm{N}}$

$\bullet \bullet \bullet \bullet \bullet \bullet \bullet \bullet \bullet \bullet \bullet \bullet \bullet \bullet \bullet \bullet$ $\bullet \bullet \bullet \bullet \bullet \bullet \bullet \bullet \bullet \bullet \bullet \bullet \bullet$

$\bullet \bullet \bullet \bullet \bullet \bullet \bullet \bullet \bullet \bullet \bullet \bullet y_{y}=2$

$\bullet \bullet \bullet \bullet \bullet \bullet \bullet \bullet \bullet \bullet \bullet \bullet \bullet y_{y}=1$

$\bullet \bullet \bullet \bullet \bullet \bullet \bullet \bullet \bullet \bullet \bullet y_{y=N}$ $\mathfrak{S}(0)$
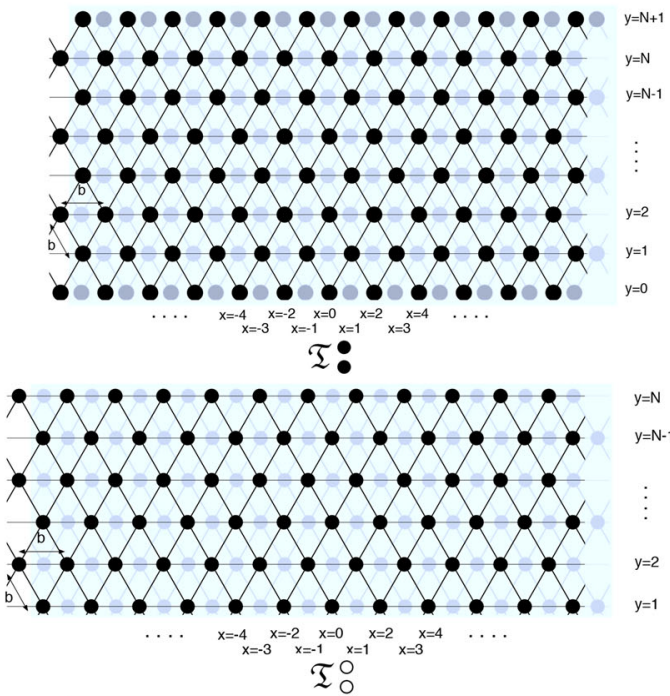

政 +1

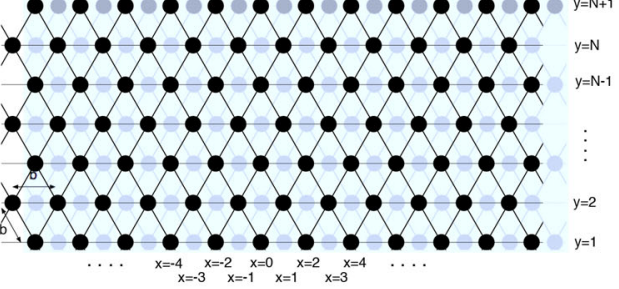

$\mathfrak{T}_{0}$
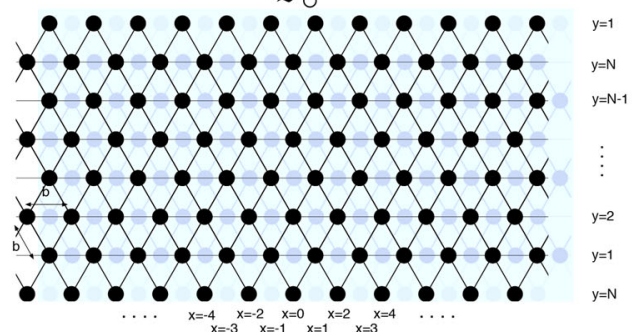

$\ldots \ldots$

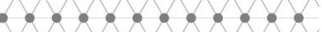
$\cdots \cdots . . . .6$

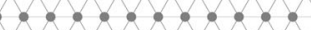

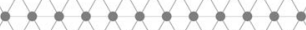
……… $\ldots 20.20$ .......

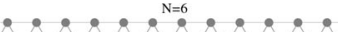

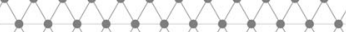

. . . . . . . .

, . . . . . . .

. . . . . . . . .

(

$\mathfrak{T}(0)$

Figure 2. Illustration of (left) square and (right) triangular lattice waveguides, $\mathfrak{\subseteq}_{N}$ and $\mathfrak{I}_{N}$, respectively, with four different types of boundary. $\mathfrak{I}_{N}$ and $\mathfrak{I}_{N}^{\mathrm{R}}$ are shown together. $\mathfrak{I}_{N}$ is also shown on the right.

$$
\begin{aligned}
M \frac{d^{2}}{d t^{2}} u_{x, y}= & K\left(u_{x+1, y+1}+u_{x+1, y-1}+u_{x-1, y+1}+u_{x-1, y-1}\right. \\
& \left.+u_{x+2, y}+u_{x-2, y}-6 u_{x, y}\right),(x, y) \in \mathbb{Z}_{N}^{2}
\end{aligned}
$$

Motivated by a three-dimensional context, let $M=\rho b^{3}, K=$ $\frac{2}{3} \mu b$ and $t=t c_{s}$. This implies that the particles have a unit mass, the equilibrium in-plane spacing between the nearest neighbours is $b$ and the nearest neighbour (anti-plane) interactions have spring constant $2 /\left(3 b^{2}\right)$. The triangular lattice dispersion relation $[52,65,81]$ is $\omega^{2}=\frac{1}{b^{2}} \Omega_{\mathfrak{I}}^{2}(\xi, \eta)$, where

$$
\begin{aligned}
\Omega_{\mathfrak{I}}^{2}(\xi, \eta)= & \frac{2}{3}\left(4 \sin ^{2} \xi+4 \sin ^{2} \frac{1}{2}(\xi+\eta)\right. \\
& \left.+4 \sin ^{2} \frac{1}{2}(\xi-\eta)\right), \quad(\xi, \eta) \in[-\pi, \pi]^{2} .
\end{aligned}
$$

Table 2. Variants of $\mathfrak{I}_{N}$.

\begin{tabular}{lcc}
\hline S. no. & Strip & Remark \\
\hline 1 & $\mathfrak{I}_{0}^{\bullet}$ & Even/odd modes \\
2 & $\mathfrak{T}_{\circ}^{\circ}$ & Even/odd modes \\
3 & $\mathfrak{T}_{\circ}^{\circ}$ & Asymmetric \\
4 & $\mathfrak{T} \odot$ & $N$ is even \\
\hline
\end{tabular}

The representative variants of the triangular lattice waveguides along with boundary conditions are listed in table 2 (see also figure 2 and Remark 1). Using the lattice model formulation, the lattice waveguides of $\mathfrak{I}$ are decorated with the symbols $\bullet$ and $\circ$ as superscript (respectively. subscript) to indicate the fixed and free upper (respectively. lower) boundary condition, respectively, and (?) to denote the periodic boundary condition. 
Remark 1 Excluding the special cases of periodic boundary $\mathfrak{S} \odot$ and $\mathfrak{I} \odot$, it is easy to see that there is one combinatorially different variant that is absent (because it is related by reflection symmetry) in tables 1 and 2 for square and triangular lattice waveguides, respectively, out of the total four such possible variants.

Remark $2 u$ is dimensionless displacement, $x$ is dimensionless horizontal position, $y$ is dimensionless vertical position and $t$ is the dimensionless time. For both lattice models, in the continuum limit, using appropriate continuous coordinates $x, y, t$, the usual wave equation can be obtained as $b / L \rightarrow 0$ (with reference to a macroscopic length scale $L$ ). The details are also available in standard texts in numerical analysis, for example, [77] (see also $[64,65])$.

In the following three sections, several waveguides with three different structures are analyzed along with a provision of the detailed derivations of the dispersion relations and corresponding wave modes. First the square lattice waveguides are considered with nearest nearest neighbor interactions only, followed by triangular lattice waveguides of the similar type, and the fourth section details the square lattice waveguides incorporating the next-nearest neighbor interactions as well. It is assumed throughout the paper that the time dependence of the propagating wave modes is described by the term $e^{-i \omega t}$ and the space dependence by $e^{i \xi x}$; moreover the former factor is suppressed. For convenience of notation (refer (2), (4)), a scaled frequency $\omega$ is defined by $\omega:=b \omega$.

\section{Waves in $\Im_{N}$}

The equation for a particle in $\Im_{N}$ located at lattice site $(x, y)$, such that it does not belong to either of the strip boundary rows, i.e., $y \notin\{1, N\}$ but $y \in \mathbb{Z}_{2}^{N}-1$, is

$$
-\omega^{2} u_{x, y}=\sum_{i \in\{-1,1\}}\left(u_{x+i, y}+u_{x, y+i}\right)-4 u_{x, y}, \quad \forall x \in \mathbb{Z} .
$$

Corresponding to the lattice waveguides listed in table 1, the boundary conditions at upper and lower rows studied in this paper $\forall x \in \mathbb{Z}$, are as follows: at $y=N$

$$
\begin{gathered}
-\omega^{2} u_{x, y}=u_{x+1, y}+u_{x-1, y}+u_{x, y-1}-4 u_{x, y} \\
-\omega^{2} u_{x, y}=u_{x+1, y}+u_{x-1, y}+u_{x, y-1}-3 u_{x, y} \\
-\omega^{2} u_{x, y}=u_{x+1, y}+u_{x-1, y}+u_{x, y-1}+u_{x, 1}-4 u_{x, y}
\end{gathered}
$$

and at $y=1$

$$
\begin{gathered}
-\omega^{2} u_{x, y}=u_{x+1, y}+u_{x-1, y}+u_{x, y+1}-4 u_{x, y} \\
-\omega^{2} u_{x, y}=u_{x+1, y}+u_{x-1, y}+u_{x, y+1}-3 u_{x, y} \\
-\omega^{2} u_{x, y}=u_{x+1, y}+u_{x-1, y}+u_{x, y+1}+u_{x, N}-4 u_{x, y}
\end{gathered}
$$

Remark 3 The expressions related to linear waves that have been derived for the square lattice waveguides are well known, though they are presented below in the language of the Chebyshev polynomials. A slightly generalized model with next-nearest-neighbour interactions is analysed later (section 5).

In the following, the four types of boundary conditions are considered for the lattice waveguides of square lattice structure (with nearest-neighbour interactions), namely, those with fixed boundary $\mathfrak{\Xi}_{0}^{\circ}$, free boundary $\mathfrak{S}_{0}^{\circ}$, fixed-free boundary $\mathfrak{S}_{\circ}^{\bullet}$ and the periodic boundary $\mathfrak{S}_{\odot}$ (see the left of figure 2 for illustrations).

\subsection{Fixed boundary $\mathfrak{\Xi}^{\bullet}$}

For the upper and lower boundary rows the conditions (6a) and (7a) hold, respectively. With the assumed space $(x)$ dependence, i.e. $u_{x, y}=a_{y} e^{i \kappa_{x} x}$, it follows that

$$
\begin{gathered}
-\omega^{2} a_{y}=\left(1-\delta_{y, N}\right) a_{y+1}+\left(1-\delta_{y, 1}\right) a_{y-1}+2 \cos \xi a_{y}-4 a_{y} \\
y \in \mathbb{Z}_{1}^{N} .
\end{gathered}
$$

Let $\mathbf{A}_{N} \in \mathbb{C}^{N \times N}$, a matrix with four perturbed corners [63], be defined by

$$
\begin{aligned}
\mathbf{A}_{N}= & {\left[\begin{array}{ccccc}
\chi & \Upsilon & 0 & \cdots & 0 \\
\Upsilon & \chi & \Upsilon & 0 & \ldots \\
0 & \Upsilon & \ddots & \ddots & 0 \\
\cdots & & \cdots & \chi & \Upsilon \\
0 & \cdots & 0 & \Upsilon & \chi
\end{array}\right] } \\
+ & {\left[\begin{array}{ccc}
\delta_{N W} & 0 \cdots 0 & \delta_{N E} \\
0 & 0 \ldots 0 & 0 \\
\vdots & \ddots & \vdots \\
0 & 0 \ldots 0 & 0 \\
\delta_{S W} & 0 \cdots 0 & \delta_{S E}
\end{array}\right] . }
\end{aligned}
$$

It is easy to see that $u$ is a propagating wave in $\mathfrak{S}_{\bullet}^{\bullet}$, with $\mathbf{A}_{N}$ of the form (9), if $a \in \mathbb{C}^{N}$ satisfies

$$
\begin{gathered}
\left(\mathbf{A}_{N}-\Lambda \mathbf{I}_{N}\right) a=0, a \in \mathbb{C}^{N}, \text { where } \\
\Lambda=\omega^{2}, \chi(\xi)=4-2 \cos \xi, \Upsilon=-1,
\end{gathered}
$$

and $\delta_{N E}=\delta_{S W}=0, \delta_{N W}=\delta_{S E}=0$. According to [61], the eigenvalue problem (10) is solved by the eigenpairs 
(a)

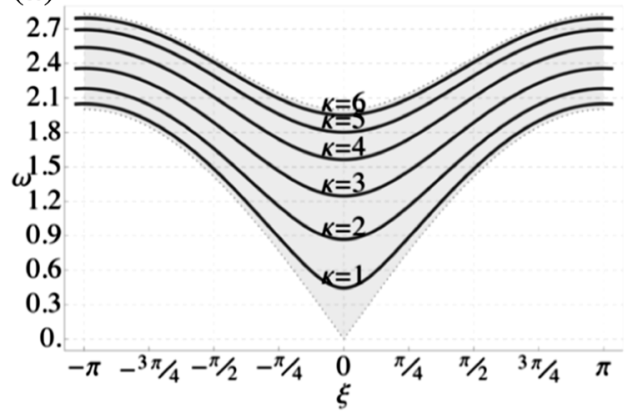

(c)

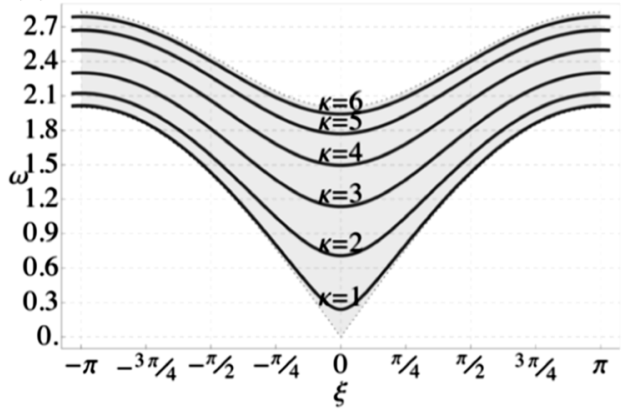

Figure 3. Dispersion relation $\Omega_{\kappa}, \kappa \in \mathbb{Z}_{1}^{N}$, with $N=6$ for $\mathfrak{S}_{N}$ : infinite lattice is illustrated by grey-coloured shaded region.

described therein; in particular, each eigenvalue $\Lambda$ is described by the general form

$$
\Lambda=\chi+2 \Upsilon \cos \eta_{\kappa}, \quad \kappa=1, \ldots, N,
$$

where $\eta_{\kappa}$ is determined by a condition depending on $\mathbf{A}_{N}$, given by (3.10) of the same paper, i.e.

$$
\begin{gathered}
\Upsilon^{2} \sin (N+1) \eta_{\kappa}+\left(\delta_{N W} \delta_{S E}-\delta_{N E} \delta_{S W}\right) \sin (N-1) \eta_{\kappa} \\
-\Upsilon\left(\delta_{N W}+\delta_{S E}\right) \sin N \eta_{\kappa}-\Upsilon\left(\delta_{N E}+\delta_{S W}\right) \sin \eta_{\kappa}=0 .
\end{gathered}
$$

Thus, in case of $\mathfrak{S}_{:}^{\bullet}$, the dispersion relations and normal modes are given by

$$
\begin{gathered}
\omega^{2}=\Omega_{\kappa}^{2}(\xi)=4 \sin ^{2} \frac{1}{2} \eta_{\kappa}+4 \sin ^{2} \frac{1}{2} \xi=\Omega_{\Im}^{2}\left(\xi, \eta_{\kappa}\right), \quad(14 \mathrm{a}) \\
a_{(\kappa) v}=\sqrt{\frac{2}{N+1}} \sin v \eta_{\kappa}, v \in \mathbb{Z}_{1}^{N}, \quad \eta_{\kappa}=\frac{\kappa \pi}{N+1}, \kappa \in \mathbb{Z}_{1}^{N} .
\end{gathered}
$$

Remark 4 Since $\mathbf{A}_{N}$ is a Hermitian matrix, the vectors $\left\{a_{(\kappa)}\right\}_{\kappa \in \mathbb{Z}_{1}^{N}}$ form an orthonormal basis for $\mathbb{C}^{N}$ (with standard inner product).

Since the lattice rows adjacent to $\boldsymbol{\Xi}_{\text {: }}^{\bullet}$ are fixed, evidently $\sin 0 \eta_{\kappa}=0$ and $\sin (N+1) \eta_{\kappa}=0$. In terms of the Chebyshev polynomials [56], the condition (13) can be expressed as

(b)

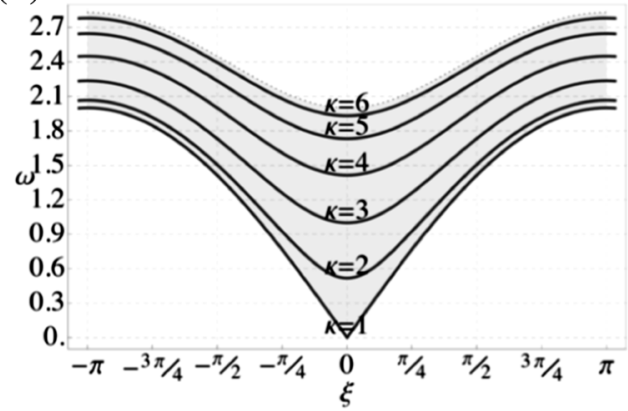

(d)

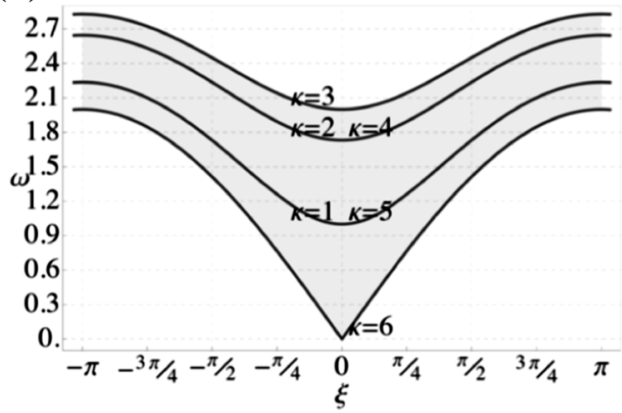

(a) $\mathfrak{S}_{:}^{:}$, (b) $\mathfrak{S}_{\circ}^{\circ}$, (c) $\mathfrak{S}_{0}^{\cdot}$ and (d) $\mathfrak{S}_{\odot}$. The pass band envelope of the . 
For convenience of obtaining the solution of this equation, it is assumed that $\mathfrak{I} \omega>0$ (allowing the limit $\omega \rightarrow 0^{+}$ whenever required). Let

$$
\mathscr{2}(\xi):=4-2 \cos \xi-\omega^{2}, \imath^{2}=\mathscr{Q}+2, h^{2}=\mathscr{Q}-2,
$$

and $\lambda$ be defined by

$$
\lambda=\frac{\imath-h}{\imath+h}
$$

and the branch cuts are chosen such that $|\lambda|<1$ on the complex plane outside the branch cuts (see [55, 64] for details, for example). Using the definition of Chebyshev polynomial of second kind [58], it follows that

$$
\lambda^{-n}-\lambda^{n}=\left(\lambda^{-1}-\lambda\right) U_{n-1}\left(\frac{1}{2}\left(\lambda+\lambda^{-1}\right)\right), \quad 0<n \in \mathbb{Z}
$$

Using (17) and (18), consider the definition

$$
\vartheta:=\vartheta(\xi)=\frac{1}{2} \mathscr{Q}(\xi), \xi \in[-\pi, \pi]
$$

Remark 5 Throughout the paper, the argument of Chebyshev polynomials $\vartheta$ is defined by (21). Further, let $\lambda=e^{i \eta}$, so that $\mathscr{Q}=2 \cos \eta$ by (17); also $\vartheta=\cos \eta$. It is easy to see that $\lambda^{-1}-\lambda=\imath h$. See [55] for many other relations and their applications.

Due to the fixed boundary condition at upper row, using (16) it is required that $C_{1} \lambda^{N+2}+C_{2} \lambda^{-N-2}=0$. Taking into account the even symmetry, $C_{1} \lambda^{2}+C_{2} \lambda^{-2}=C_{1}+C_{2}$. For a non-trivial solution $C_{1}, C_{2}$ of the two (linear algebraic) equations, it is required that $\left(1-\lambda^{-2}\right) \lambda^{N+2}-(1-$ $\left.\lambda^{2}\right) \lambda^{-N-2}=0$. Using the Chebyshev polynomials $U_{n}$ of second kind (20), it is found that

$$
U_{N+1}(\vartheta)-U_{N-1}(\vartheta)=0 .
$$

Using the Chebyshev polynomials $T_{n}$ of first kind, (22a) can be re-written as $T_{N+1}(\vartheta)=0$, where $\vartheta=\cos \eta_{\kappa}$. Hence, using the well-known roots of the Chebyshev polynomials [58], $\quad \cos \eta_{\kappa}=\cos \left(\kappa-\frac{1}{2}\right) \pi /(N+1), \quad \kappa \in$ $\mathbb{Z}_{1}^{N+1}$, same as those stated earlier $(14 \mathrm{~b})$ for odd $\kappa$. Similarly, when $N=2 N$, for the even modes, $C_{1} \lambda^{N+1}+$ $C_{2} \lambda^{-N-1}=0$, and $C_{1} \lambda+C_{2} \lambda^{-1}=C_{1}+C_{2}$. For a nontrivial solution $C_{1}, C_{2}$ of the two (linear algebraic) equations, it is required that $\left(1-\lambda^{-1}\right) \lambda^{N+1}-(1-\lambda) \lambda^{-N-1}=$ 0 . Using the Chebyshev polynomials $U_{n}$ of second kind (after the application of (20)), it is found that

$$
U_{N}(\vartheta)-U_{N-1}(\vartheta)=0
$$

Using the Chebyshev polynomials $V_{n}$ of third kind, this can be re-written as $V_{N}(\vartheta)=0$, where $\vartheta=\cos \eta_{\kappa}$, which implies [58] that $\cos \eta_{\kappa}=\cos \left(\kappa-\frac{1}{2}\right) \pi /\left(N+\frac{1}{2}\right), \kappa \in \mathbb{Z}_{1}^{N}$, same as those stated earlier (14b) for odd $\kappa$.

For the odd modes in case of $N=2 N+1$, it is found that

$$
U_{N}(\vartheta)=0
$$

which implies [58] that $\cos \eta_{\kappa}=\cos \kappa \pi /(N+1), \kappa \in \mathbb{Z}_{1}^{N}$, same as those stated earlier (14b) for even $\kappa$. When $N=2 N$ and the odd modes are considered, then analogous to (22a), the condition can be found as

$$
U_{N}(\vartheta)+U_{N-1}(\vartheta)=0
$$

where $\vartheta$ is given by (21). Using the Chebyshev polynomials $W_{n}$ of fourth kind, this can be re-written as $W_{N}(\vartheta)=$ $0, \vartheta=\cos \eta_{\kappa} \quad$ yielding $\cos \eta_{\kappa}=\cos \kappa \pi /\left(N+\frac{1}{2}\right), \quad \kappa \in \mathbb{Z}_{1}^{N}$, same as those stated earlier in (14b) for even values of $\kappa$.

\subsection{Free boundary $\mathfrak{\subseteq}_{\circ}^{\circ}$}

For $\mathfrak{\Xi}_{\circ}^{\circ}$, i.e., $\mathfrak{\Xi}_{N}$ with a free upper and lower boundary, the equations (6b) and (7b) hold, respectively. Again, based on an equation analogous to (8), it is found that (10) holds for the normal modes, due to the same reasons as cited for $\mathfrak{S}_{\bullet}^{\bullet}$, where $\mathbf{A}_{N}$ has the form (9) with (11) and $\delta_{N E}=\delta_{S W}=0, \delta_{N W}=\delta_{S E}=-1$. Thus, in case the boundary rows of $\Xi_{N}$ are free, the dispersion relations (14a) are obtained with the corresponding normal modes and $\eta_{\kappa}$ given by

$a_{(\kappa) v}=\sqrt{\frac{2}{N}}\left\{\begin{array}{cc}\cos \left(v-\frac{1}{2}\right) \eta_{\kappa}, & \kappa \neq 1 \\ \frac{1}{\sqrt{2}}, & \kappa=1\end{array}, v \in \mathbb{Z}_{1}^{N}, \quad \eta_{\kappa}=\frac{(\kappa-1) \pi}{N}, \kappa \in \mathbb{Z}_{1}^{N}\right.$.

Indeed, using (13), $\sin (N+1) \eta_{\kappa}+\sin (N-1) \eta_{\kappa}-2$ $\sin N \eta_{\kappa}=0$, i.e., in terms of the Chebyshev polynomials of second kind (20),

$$
2(\vartheta-1) U_{N-1}(\vartheta)=0,
$$

which implies $\vartheta=\cos (\kappa-1) \pi / N, \kappa \in \mathbb{Z}_{1}^{N}$, the same as stated in $(24)_{2}$. The expression (25) has been plotted and shown in figure $3 \mathrm{~b}$.

The odd modes correspond to even values of $\kappa$, while the even modes correspond to odd values of $\kappa$. For instance, when $N=2 N$ and odd modes are considered, then (since $\kappa \neq 1)$ using $\cos \left(v-\frac{1}{2}\right) \eta_{\kappa}=-\cos \left(2 N+1-v-\frac{1}{2}\right) \eta_{\kappa}=$ $(-1)^{\kappa} \cos \left(v-\frac{1}{2}\right) \eta_{\kappa}$, it is found that $\kappa$ is even. Similar reasoning holds for even modes.

By an application of the second method, as described in section 3.1, the same expressions, as stated earlier, can be 
also derived. For even modes in $\mathfrak{\subseteq}_{\circ}^{\circ}$ with $N=2 N+1$, it is required that $a_{v}=a_{2 N+2-v}$. Using (16), taking into account the symmetry and free boundary condition, for non-trivial $C_{1}, C_{2}$, it is easily found that $\left(1-\lambda^{-2}\right)\left(\lambda^{N+2}-\lambda^{N+1}\right)-$ $\left(1-\lambda^{2}\right)\left(\lambda^{-N-2}-\lambda^{-N-1}\right)=0$. Using the Chebyshev polynomials $U_{n}$ of second kind (after an application of (20))

$$
U_{N+1}(\vartheta)-U_{N-1}(\vartheta)-\left(U_{N}(\vartheta)-U_{N-2}(\vartheta)\right)=0,
$$

where $\vartheta$ is given by (21). Using (102a) $)_{1}$ and the Chebyshev polynomials $W_{n}$ of fourth kind, (26a) is expressed as $2(\vartheta-$ 1) $W_{N}(\vartheta)=0$, with $\vartheta=\cos \eta_{\kappa}$. Hence, it is found that $\eta_{\kappa}=$ $\kappa \pi /\left(N+\frac{1}{2}\right), \kappa \in \mathbb{Z}_{0}^{N}$, and $\eta_{\kappa}$ is an even multiple of $\pi / N$, i.e., the same values of $\eta_{\kappa}$ as earlier above in (24) for odd values of $\kappa$. Similarly, for $N=2 N$, using the general solution

$$
a_{N+v}=C_{1} \lambda^{v}+C_{2} \lambda^{-v}, v \in \mathbb{Z}_{1}^{N},
$$

for the even modes, it is found that $\left(1-\lambda^{-1}\right)\left(\lambda^{N+1}-\right.$ $\left.\lambda^{N}\right)-(1-\lambda)\left(\lambda^{-N-1}-\lambda^{-N}\right)=0$, i.e., $2 U_{N-1}(\vartheta)-U_{N}(\vartheta)$ $-U_{N-2}(\vartheta)=0$, which can written as

$$
2(\vartheta-1) U_{N-1}(\vartheta)=0 .
$$

This implies that $\eta_{\kappa}=\kappa \pi / N, \kappa \in \mathbb{Z}_{0}^{N}$. Hence, $\eta_{\kappa}$ is an even multiple of $\pi / N$, i.e. the same values of $\eta_{\kappa}$ as stated earlier in (24) for odd values of $\kappa$. When $N=2 N$, for the odd modes, analogous to (26a),

$$
U_{N}(\vartheta)-U_{N-2}(\vartheta)=0,
$$

where $\vartheta$ is given by (21). Using the Chebyshev polynomials $T_{n}$ of first kind, this can be re-written as $T_{N}(\vartheta)=0, \vartheta=\cos \eta_{\kappa}$, yielding $\cos \eta_{\kappa}=\cos \left(\kappa-\frac{1}{2}\right) \pi / N$, $\kappa \in \mathbb{Z}_{1}^{N}$, the same as those stated above in (24) for even $\kappa$. For the odd modes in case of $N=2 N+1$, taking into account the symmetry and free boundary condition, it is easily found that (20) $\left(\lambda^{N+1}-\lambda^{N}\right)-\left(\lambda^{-N-1}-\lambda^{-N}\right)=0$, i.e.

$$
U_{N}(\vartheta)-U_{N-1}(\vartheta)=0 .
$$

Using the Chebyshev polynomials $V_{n}$ of third kind, $V_{N}(\vartheta)=0, \vartheta=\cos \eta_{\kappa}$, which implies that $\cos \eta_{\kappa}=$ $\cos \left(\kappa-\frac{1}{2}\right) \pi /\left(N+\frac{1}{2}\right), \kappa \in \mathbb{Z}_{1}^{N}$. Hence, $\eta_{\kappa}$ is an odd multiple of $\pi / N$, i.e., the same values of $\eta_{\kappa}$ as stated earlier in (24) for even values of $\kappa$.

\subsection{Fixed-free boundary $\mathfrak{\Xi}_{\text {。 }}^{\bullet}$}

Consider the waveguide $\mathfrak{S}_{0}^{\cdot}$, as shown schematically in the left side of figure 2. The equation of motion for the lattice row at $y=1$ is (6a), corresponding to a fixed boundary, while at $y=N$ it is (7b), corresponding to a free boundary. Hence, based on an equation analogous to (8), it is found that (10) holds for the normal modes, where $\mathbf{A}_{N}$ has the form (9) with (11) and $\delta_{N E}=\delta_{S W}=0, \delta_{N W}=0, \delta_{S E}=-1$. Thus, in the present case when the boundary rows are fixed-free, using (13), $\sin (N+1) \eta_{\kappa}-\sin N \eta_{\kappa}=0$, as a consequence of (20) this yields

$$
U_{N}(\vartheta)-U_{N-1}(\vartheta)=0 .
$$

Using the Chebyshev polynomials $V_{n}$ of third kind, this implies that $V_{N}(\vartheta)=0, \vartheta=\cos \eta_{\kappa}$, so that $\vartheta=\cos \left(\kappa-\frac{1}{2}\right) \pi /\left(N+\frac{1}{2}\right), \kappa \in \mathbb{Z}_{1}^{N}$. Thus, the dispersion relation is (14a) and the corresponding normal modes are

$$
\begin{aligned}
a_{(\kappa) v} & =\sqrt{\frac{4}{2 N+1}} \cos \left(v-\frac{1}{2}\right) \eta_{\kappa}, v \in \mathbb{Z}_{1}^{N}, \\
\eta_{\kappa} & =\frac{(2 \kappa-1) \pi}{2 N+1}, \kappa \in \mathbb{Z}_{1}^{N} .
\end{aligned}
$$

In the free-fixed case, i.e. $\mathfrak{S}_{\bullet}^{\circ}$, it is easy to find that $a_{(\kappa) v}=$ $\sqrt{4 /(2 N+1)} \sin v \eta_{\kappa}, v \in \mathbb{Z}_{1}^{N}$. The expression (28) has been plotted and shown in figure 3c. An application of the second method, as described in sections 3.1 and 3.2, yields the same expressions as obtained earlier.

\subsection{Periodic boundary $\Subset \odot$}

Due to the periodic upper and lower boundary rows, (6c) and (7c) hold. Based on an equation analogous to (8), it follows that (10) holds, where $\mathbf{A}_{N}$ has the form (9) with (11) and $\delta_{N E}=\delta_{S W}=\Upsilon, \delta_{N W}=\delta_{S E}=0$; in particular, it is a circulant matrix for which the eigenpairs are straightforward to find [60]. The normal modes satisfy the dispersion relation (14a) with

$$
a_{(\kappa) v}=\frac{1}{\sqrt{N}} e^{i v \eta_{\kappa}}, v \in \mathbb{Z}_{1}^{N}, \quad \eta_{\kappa}=\frac{2 \kappa \pi}{N}, \kappa \in \mathbb{Z}_{1}^{N} .
$$

An alternative derivation based on [61] is the following. From (13), the dispersion relation is given by $\omega^{2}=\Omega_{\kappa}^{2}$ with (12) and $\eta_{\kappa}$ such that $\Upsilon^{2} \sin (N+1) \eta_{\kappa}-\Upsilon^{2} \sin (N-1) \eta_{\kappa}-$ $2 \Upsilon^{2} \sin \eta_{\kappa}=0$, i.e.

$$
U_{N}(\vartheta)-U_{N-2}(\vartheta)-2=0 .
$$

It is easy to see that $(30)_{2}$ describes the roots that satisfy this condition. By an application of the results of [61], the eigenvectors $a_{(\kappa) v}$ can be also expressed as linear combinations of $\sin v \eta_{\kappa}$ and $\cos v \eta_{\kappa}$ for $v \in \mathbb{Z}_{1}^{N}$, equivalent to (30). The expression (31) has been plotted and shown in figure $3 \mathrm{~d}$ by marking the doubly repeated dispersion relations (except for the uppermost and lowermost). An application of the second method (i.e., using $\lambda$ ), as described in sections 3.1 and 3.2, yields the same expressions. 
Remark 6 Note that there are no surface modes, i.e., localized propagating waves, in any of the square lattice waveguides listed in table 1. Also, the Remark 4 holds for the normal modes of all square lattice waveguides stated in table 1. See Appendix 2.1 for the proof of normalization that appears in (14b), (24) and (29).

The following section presents the analysis, for the entire collection of four types of boundary conditions, similar to the earlier one for the lattice waveguides with triangular structure.

\section{Waves in $\mathfrak{I}_{N}$}

The equation for particles in $\mathfrak{I}_{N}$, away from the boundary rows, is

$$
\begin{aligned}
-\frac{3}{2} \omega^{2} u_{x, y} & =u_{x+2, y}+u_{x-2, y}+u_{x-1, y+1}+u_{x-1, y+1}+u_{x+1, y-1} \\
& +u_{x-1, y-1}-6 u_{x, y}, \quad \forall x \in \mathbb{Z}, y \in \mathbb{Z}_{2}^{N-1} .
\end{aligned}
$$

The different pairs of boundary conditions leading to the symbolic arrangements listed in table 2 involve the following: $\forall x \in \mathbb{Z}, y=N$

$$
\begin{gathered}
-\frac{3}{2} \omega^{2} u_{x, y}=u_{x+2, y}+u_{x-2, y}+u_{x+1, y-1}+u_{x-1, y-1}-6 u_{x, y} \\
-\frac{3}{2} \omega^{2} u_{x, y}=u_{x+2, y}+u_{x-2, y}+u_{x+1, y-1}+u_{x-1, y-1}-4 u_{x, y} \\
-\frac{3}{2} \omega^{2} u_{x, y}=u_{x+2, y}+u_{x-2, y}+u_{x+1, y-1}+u_{x-1, y-1} \\
+u_{x+1, y-N+1}+u_{x-1, y-N+1}-6 u_{x, y}
\end{gathered}
$$

and $\forall x \in \mathbb{Z}, y=1$

$$
-\frac{3}{2} \omega^{2} u_{x, y}=u_{x+2, y}+u_{x-2, y}+u_{x+1, y+1}+u_{x-1, y+1}-6 u_{x, y}
$$

$$
-\frac{3}{2} \omega^{2} u_{x, y}=u_{x+2, y}+u_{x-2, y}+u_{x+1, y+1}+u_{x-1, y+1}-4 u_{x, y} \quad \text { (०), }
$$

$$
\begin{aligned}
-\frac{3}{2} \omega^{2} u_{x, y}= & u_{x+2, y}+u_{x-2, y}+u_{x+1, y+1} \\
& +u_{x-1, y+1}+u_{x+1, y+N-1}+u_{x-1, y+N-1}-6 u_{x, y}
\end{aligned}
$$

Remark 7 The expressions derived later for the triangular lattice waveguides have not all been studied earlier, especially the case of waveguide with one or both boundary conditions free; see an application of the fixed boundary condition by Sharma [24].

In the following, the four types of boundary conditions, namely fixed boundary $\mathfrak{I}_{\bullet}^{\bullet}$, free boundary $\mathfrak{I}_{\circ}^{\circ}$, fixed-free boundary $\mathfrak{I}_{\circ}^{\bullet}$ and the periodic boundary $\mathfrak{I} \odot$, are considered (see the right of figure 2 for illustrations).

\subsection{Fixed boundary $\mathfrak{I}^{\bullet}$}

For this case, at $y=N$, (33a) holds while for $y=1$, (34a) holds corresponding to either of the fixed boundaries of $\mathfrak{I}_{\bullet}^{\bullet}$. With the representation of a propagating wave in $\mathfrak{I}_{\bullet}^{\bullet}$ as the ansatz $u_{x, y}=a_{y} e^{i \kappa_{x} x}$, it is found that

$$
\begin{aligned}
-\frac{3}{2} \omega^{2} a_{y}= & \left(1-\delta_{y, N}\right) 2 \cos \xi a_{y+1}+\left(1-\delta_{y, 1}\right) 2 \cos \xi a_{y-1} \\
& +2 \cos 2 \xi a_{y}-6 a_{y}, \quad y \in \mathbb{Z}_{1}^{N} .
\end{aligned}
$$

Equivalently, $a \in \mathbb{C}^{N}$ satisfies (10) where $\mathbf{A}_{N}$ has the form (9) with

$$
\Lambda=\frac{3}{2} \omega^{2}, \chi(\xi)=6-2 \cos 2 \xi, \Upsilon(\xi)=-2 \cos \xi,
$$

and $\delta_{N E}=\delta_{S W}=0, \delta_{N W}=\delta_{S E}=0 . \quad$ Applying $\quad$ (13), $\sin (N+1) \eta_{\kappa}=0$ or $U_{N}(\vartheta)=0, \vartheta=\cos \eta_{\kappa}$. Thus, in this case when the boundary rows are fixed (i.e., $a_{0}=0, a_{N+1}=0$ ), for $\kappa \in \mathbb{Z}_{1}^{N}$, the dispersion relations and the corresponding eigemodes are given by

$$
\frac{3}{2} \omega^{2}=\frac{3}{2} \Omega_{\kappa}^{2}(\xi)=6-2 \cos 2 \xi-4 \cos \xi \cos \eta_{\kappa}=\frac{3}{2} \Omega_{\mathfrak{I}}^{2}\left(\xi, \eta_{\kappa}\right),
$$

$a_{(\kappa) v}=\sqrt{\frac{2}{N+1}} \sin v \eta_{\kappa}, v \in \mathbb{Z}_{1}^{N}, \quad \eta_{\kappa}=\frac{\kappa \pi}{N+1}, \kappa \in \mathbb{Z}_{1}^{N}$.

The odd modes correspond to even values of $\kappa$, while the even modes correspond to odd values of $\kappa$; the detailed reasoning is the same as that for $\mathfrak{\Xi}_{\text {: }}$ (section 3.1 ). The relations (37) have been graphically illustrated in figure $4 \mathrm{a}$.

Remark 8 Let $N=2 N$. Notice that, with $\widetilde{\xi}=\pi-\xi$,

$$
\begin{aligned}
\frac{3}{2} & \Omega_{\kappa}^{2}(\widetilde{\xi})=6-2 \cos 2 \widetilde{\xi}-4 \cos \widetilde{\xi} \cos \eta_{\kappa}=6-2 \cos 2 \xi \\
& -4 \cos \xi \cos \frac{2 N+1-\kappa}{2 N+1} \pi,
\end{aligned}
$$

and when $\kappa$ is odd (respectively even), $2 N+1-\kappa$ is even (respectively odd). This implies that the dispersion curves for the odd and even modes do not have symmetry about the point corresponding to $\xi= \pm \frac{1}{2} \pi$. However, with $N=2 N+1$, since $\kappa$ odd (respectively even) is equivalent to $2 N+2-\kappa$ odd (respectively even), the dispersion 
(a)

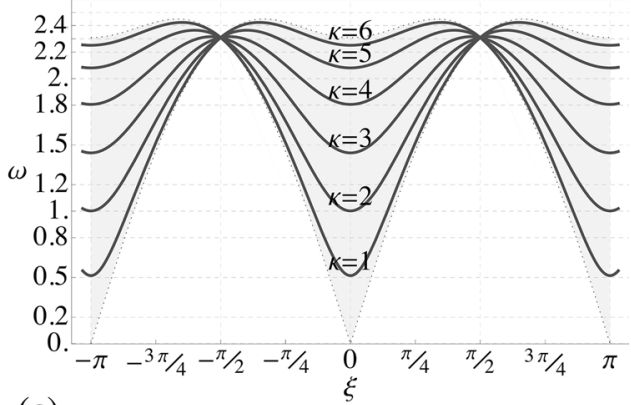

(c)

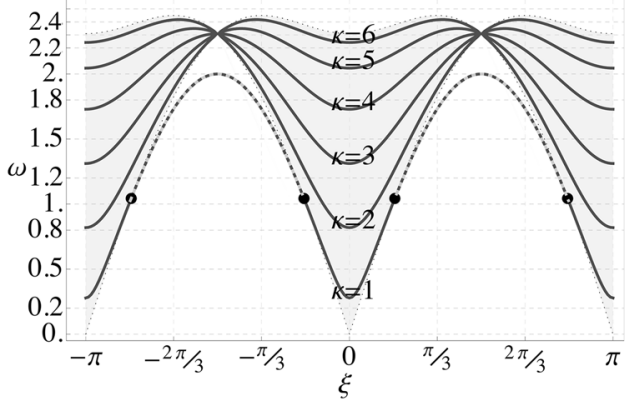

(b)

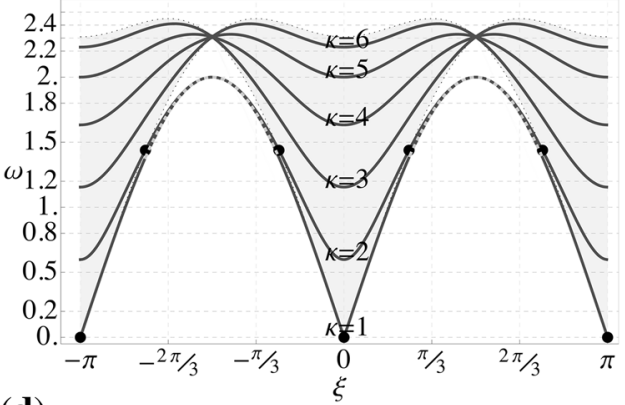

(d)

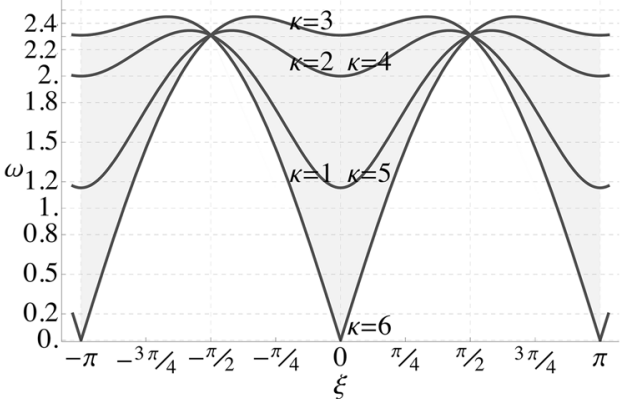

Figure 4. Dispersion relation $\Omega_{\kappa}, \kappa \in \mathbb{Z}_{1}^{N}$, with $N=6$ for $\mathfrak{I}_{N}:(\mathbf{a}) \mathfrak{I}_{\bullet}^{\bullet},(\mathbf{b}) \mathfrak{I}_{\circ}^{\circ}$, (c) $\mathfrak{I}_{\circ}^{\bullet}$ and (d) $\mathfrak{I} \odot$. The dashed curves, connecting the black dots, correspond to the surface wave dispersion curves in (b) and (c). The pass band envelope of the infinite lattice is illustrated by grey-coloured shaded region.

curves for the odd and even modes are symmetric about $\xi= \pm \frac{1}{2} \pi$.

\subsection{Free boundary $\mathfrak{T}_{\circ}^{\circ}$}

In this lattice waveguide, both boundaries are free so that for $y=N$, (33b) holds while for $y=1$, (34b) holds. Based on an equation analogous to (35), it is easy to see that the normal modes are required to satisfy (10), where $\mathbf{A}_{N}$ has the form (9) with definitions provided in (36) and $\delta_{N E}=\delta_{S W}=$ $0, \delta_{N W}=\delta_{S E}=-2$. Applying the results of [61] and using (13), in case of $\mathfrak{T}_{0}^{\circ}$, it is found that the dispersion relations are given by (37) where $\eta_{\kappa}$ is such that for $\kappa \in \mathbb{Z}_{1}^{N}$, $\cos ^{2} \xi \sin (N+1) \eta_{\kappa}+\sin (N-1) \eta_{\kappa}-2 \cos \xi \sin N \eta_{\kappa}=0$. Indeed, using the Chebyshev polynomials $U_{n}$ of second kind, the latter is re-written as

$$
\cos ^{2} \xi U_{N}(\vartheta)+U_{N-2}(\vartheta)-2 \cos \xi U_{N-1}(\vartheta)=0 .
$$

Using (37), it is clear that $\eta_{\kappa}$ is a function of $\xi$ such that

$$
\cos \eta_{\kappa}=\vartheta=\frac{1}{2} \frac{6-2 \cos 2 \xi-\frac{3}{2} \omega^{2}}{2 \cos \xi} .
$$

By the nature of the expression (40), it is noted that $\eta_{\kappa}$ is allowed to assume complex values. Indeed, $\eta_{\kappa}(\xi)$ is real provided it does not correspond to a surface mode (localized wave along $y$ ) as described in section 4.2c. In general, it is assumed that $\xi \neq \pm \frac{1}{2} \pi$, while in the special case of $\xi=$ $\pm \frac{1}{2} \pi$, (39) can be used directly to calculate $\eta_{\kappa}$. Notice that unlike the expression (24) for the analogous case of $\mathfrak{\subseteq}_{\circ}^{\circ}$, (39) does not admit a closed-form expression for $\eta_{\kappa}$. Once $\eta_{\kappa}$ is determined (numerically in this case) that satisfies Eq. (39) for given $\xi$ and $\kappa \in \mathbb{Z}_{1}^{N}$, the eigenvalue $\Lambda$ of $\mathbf{A}_{N}$ is given by (12) (and (36)) and thus the dispersion relations are determined with $\Lambda=\frac{3}{2} \Omega_{\kappa}^{2}(\xi)=\frac{3}{2} \Omega_{\mathfrak{I}}^{2}\left(\xi, \eta_{\kappa}\right)$. Applying the results of [61], the eigenvectors may be found for $\kappa \in \mathbb{Z}_{1}^{N}$, as a nontrivial constant multiple of the vector with components $-\cos \xi \sin v \eta_{\kappa}+\sin (v-1) \eta_{\kappa}$. The same can be normalized (as described in Appendix 2.2) to obtain, for $\kappa \in \mathbb{Z}_{1}^{N}$,

$a_{(\kappa) v}=\frac{2\left(-\cos \xi \sin v \eta_{\kappa}+\sin (v-1) \eta_{\kappa}\right)}{\sqrt{2 N\left(1+\cos ^{2} \xi-2 \vartheta \cos \xi\right)-2+2 \cos ^{2} \xi}}, \quad v \in \mathbb{Z}_{1}^{N}$.

Instead of solving for $\eta_{\kappa}$, alternatively, $\Omega_{\kappa}^{2}$ can be also obtained directly from (39). In particular, the function $\omega^{2}=$ $\Omega_{\kappa}^{2}(\xi)$ is implicitly given by

$$
\begin{aligned}
\frac{1}{4} & \prod_{j=1}^{N}\left(6-2 \cos 2 \xi-\frac{3}{2} \Omega_{\kappa}^{2}-4 \cos \xi \cos \frac{j \pi}{N+1}\right) \\
& +\prod_{j=1}^{N-2}\left(6-2 \cos 2 \xi-\frac{3}{2} \Omega_{\kappa}^{2} 2-4 \cos \xi \cos \frac{j \pi}{N-1}\right) \\
& -\prod_{j=1}^{N-1}\left(6-2 \cos 2 \xi-\frac{3}{2} \Omega_{\kappa}^{2}-4 \cos \xi \cos \frac{j \pi}{N}\right)=0 .
\end{aligned}
$$


(b)

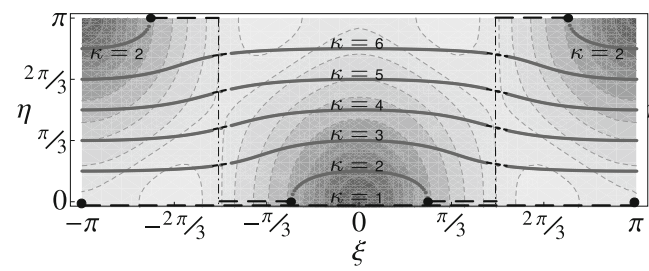

(c)

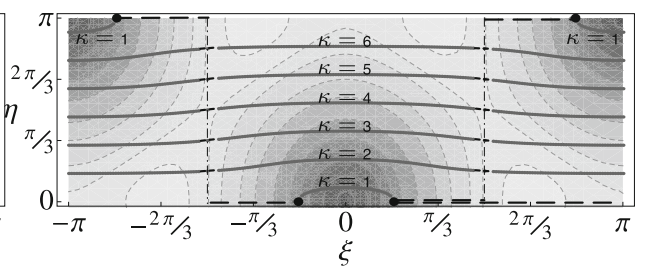

Figure 5. Contour plot of the triangular lattice dispersion relation $\Omega_{\mathfrak{I}}(\xi, \eta)$ and $\Omega_{\kappa}, \kappa \in \mathbb{Z}_{1}^{N}$, for $\mathfrak{I}_{\bullet}^{\bullet}$ (left) and $\mathfrak{I}_{\circ}^{\bullet}$ (right) with thick curves corresponding to the curves shown in figure $4 \mathrm{~b}$ and $\mathrm{c}$, respectively. Notice the gaps (marked by dashed segments) due to the surface wave dispersion curves.

The index $\kappa$ is placed in such a way that $\Omega_{\kappa}(0)$ is an increasing sequence with respect to $\kappa$. figure $4 \mathrm{~b}$ and the left of figure 5 illustrate the dispersion curves for this case.

4.2a Odd modes: For $N=2 N$, by definition of an odd mode, it is required that $a_{v}=-a_{2 N+1-v}$, i.e., the problem is equivalent to finding the eigenvectors of (10) where $\mathbf{A}_{N}$ has the form (9) with definitions provided in (36) and $\delta_{N E}=$ $\delta_{S W}=0, \delta_{N W}=-2, \delta_{S E}=2 \cos \xi$. This results in the condition

$$
\cos \xi\left(U_{N}(\vartheta)+U_{N-1}(\vartheta)\right)-U_{N-1}(\vartheta)-U_{N-2}(\vartheta)=0 .
$$

This can be also expressed in terms of the Chebyshev polynomials [58] of fourth kind $W$, leading to $\cos \xi W_{N}(\vartheta)-W_{N-1}(\vartheta)=0, \vartheta=\cos \eta_{\kappa}$. The eigenvectors are given by (41) with suitable modification for a normalized odd mode. Circumventing the results stated by [61], an alternative and direct derivation of (43) is also possible using 'the second method' (as elaborated earlier in the case of $\mathfrak{\Xi}_{N}$ ); this is presented later.

Using the equation analogous to (35), it is easy to see that the general solution $[65,82]$ for normal modes is given by (26b), where $\lambda$ satisfies

$$
\lambda+\lambda^{-1}=\mathscr{Q}(\xi):=(2 \cos \xi)^{-1}\left(6-2 \cos 2 \xi-\frac{3}{2} \omega^{2}\right) .
$$

Note that (21) also holds due to (44) and (40). Again the expressions $\iota^{2}=\mathscr{2}+2, h^{2}=\mathscr{Q}-2$ hold and $\lambda$ is given by (19). Due to free boundary condition, it is required that $2 C_{1} \lambda^{N}+2 C_{2} \lambda^{-N}=2 \cos \xi\left(C_{1} \lambda^{N+1}+C_{2} \lambda^{-N-1}\right)$. Taking into account the symmetry, it is also required that $C_{1} \lambda+$ $C_{2} \lambda^{-1}=-C_{1}-C_{2}$. For a non-trivial solution $C_{1}, C_{2}$ of both equations, it is required that $(1-\lambda \cos \xi)(1+$ $\left.\lambda^{-1}\right) \lambda^{N}-\left(1-\lambda^{-1} \cos \xi\right)(1+\lambda) \lambda^{-N}=0$. Using the Chebyshev polynomials $U_{n}$ of second kind (after an application of (20)), it is easy to see that (43) follows, where $\vartheta$ is given by (21) and (44). The eigenvectors are also, consequently, described by the expression

$$
\begin{aligned}
a_{N+v} & =-a_{(\kappa) N-v+1}=C_{N} \cos \left(v-\frac{1}{2}\right) \eta_{\kappa}, v \in \mathbb{Z}_{1}^{N}, \quad \text { where } \\
C_{N}^{-2} & =N+(\vartheta+1) \frac{\sin ^{2} \frac{1}{2} \xi}{\cos ^{2} \frac{1}{2} \xi} U_{N-1}^{2}(\vartheta),
\end{aligned}
$$

$\eta_{\kappa}, \vartheta$ satisfy (43), and $\vartheta$ is given by (21). The normalization in (45) is detailed in Appendix 2.2.

For $N=2 N+1$ and odd modes, it is required that $a_{v}=a_{2 N+2-v}$. Using the ansatz (16), the equations, based on free boundary condition and the symmetry, admit a nontrivial $C_{1}, C_{2}$, provided $(1-\lambda \cos \xi)\left(1+\lambda^{-2}\right) \lambda^{N+1}-(1-$ $\left.\lambda^{-1} \cos \xi\right)\left(1+\lambda^{2}\right) \lambda^{-N-1}=0$. Using the Chebyshev polynomials $U_{n}$ of second kind (after an application of (20)), it follows that

$$
\cos \xi U_{N}(\vartheta)-U_{N-1}(\vartheta)=0,
$$

where $\vartheta$ is given by (21). Referring to the expressions derived in section 4.3, (46) is the same as that in the case of $\mathfrak{I}^{\bullet}$ of width $N$, as intuitively expected ${ }^{2}$. The eigenvectors corresponding to the dispersion relations, based on the value of $\eta_{\kappa}$ satisfying (46), are given by a nontrivial constant multiple of the vector with components (41) or, in simpler form, for $\kappa \in \mathbb{Z}_{1}^{N}$,

$$
\begin{aligned}
a_{(\kappa) N+1+v} & =C_{N} \sin v \eta_{\kappa}, a_{(\kappa) v+1}=-a_{(\kappa) 2 N+1-v}, v \in \mathbb{Z}_{0}^{N}, \quad \text { where } \\
C_{N}^{-2} & =N+\frac{1}{2}-\frac{1}{2} \sin ^{2} \xi U_{N}^{2}(\vartheta) .
\end{aligned}
$$

See the derivation of the normalization in Appendix 2.2.

$4.2 \mathrm{~b}$ Even modes: For even modes in $\mathfrak{I}_{\circ}^{\circ}$ with $N=2 N$, it is required that $a_{v}=a_{2 N+1-v}$. The problem of finding propagating waves is equivalent to finding the eigenvectors of (10) where $\mathbf{A}_{N}$ has the form (9) with definitions provided in (36) and $\delta_{N E}=\delta_{S W}=0, \delta_{N W}=-2, \delta_{S E}=-2 \cos \xi$. After the application of the results stated in [61] (recalled as (12) and (13)), it is found that

\footnotetext{
2 Alternatively, the same expression results using (10) where $\mathbf{A}_{N+1}$ has the form (9) with definitions provided in (36) and $\delta_{N E}=\delta_{S W}=0, \delta_{N W}=-2, \delta_{S E}=0$.
} 


$$
\cos \xi\left(U_{N}(\vartheta)-U_{N-1}(\vartheta)\right)-U_{N-1}(\vartheta)+U_{N-2}(\vartheta)=0 .
$$

Using the Chebyshev polynomials $V_{n}$ of third kind, the condition (48) can also be expressed as $\cos \xi V_{N}(\vartheta)-V_{N-1}(\vartheta)=0, \vartheta=\cos \eta_{\kappa}$. The eigenvectors are given by a nontrivial constant multiple of the vector with components (41). An alternative derivation of the dispersion relation using the second method is included here as well. Using the definition of $\lambda$ in (20) and (44), the general solution for normal modes is given by (26b). Due to free-boundary condition, it is required that $2 C_{1} \lambda^{N}+$ $2 C_{2} \lambda^{-N}=2 \cos \xi\left(C_{1} \lambda^{N+1}+C_{2} \lambda^{-N-1}\right) . \quad$ Taking into account the symmetry, it is also required that $C_{1} \lambda+$ $C_{2} \lambda^{-1}=C_{1}+C_{2}$. For a non-trivial solution $C_{1}, C_{2}$ of both equations, it is necessary that $(1-\lambda \cos \xi)\left(1-\lambda^{-1}\right) \lambda^{N}-$ $\left(1-\lambda^{-1} \cos \xi\right)(1-\lambda) \lambda^{-N}=0$. Using the Chebyshev polynomials $U_{n}$ of second kind (after an application of (20)), it is found that (48) follows. The eigenvectors are described by the expression

$$
\begin{aligned}
& \qquad \begin{aligned}
a_{N+v} & =a_{(\kappa) N-v+1}=C_{N} \cos \left(v-\frac{1}{2}\right) \eta_{\kappa}, v \in \mathbb{Z}_{1}^{N}, \\
\text { where } C_{N}^{-2} & =N+(\vartheta-1) \frac{\cos ^{2} \frac{1}{2} \xi}{\sin ^{2} \frac{1}{2} \xi} U_{N-1}^{2}(\vartheta),
\end{aligned}
\end{aligned}
$$

$\eta_{\kappa}, \vartheta$ satisfy (48). The details for the normalization are provided in Appendix 2.2. For even modes in $\mathfrak{T}_{\circ}^{\circ}$ with $N=2 N+1$, it is required that $a_{v}=a_{2 N+2-v}$. Using (16), the free-boundary condition and the symmetry, non-trivial $C_{1}, C_{2}$ exist provided $(1-\lambda \cos \xi)\left(1-\lambda^{-2}\right) \lambda^{N+1}-(1-$ $\left.\lambda^{-1} \cos \xi\right)\left(1-\lambda^{2}\right) \lambda^{-N-1}=0$. Using the Chebyshev polynomials $U_{n}$ of second kind (20)

$$
\cos \xi\left(U_{N+1}(\vartheta)-U_{N-1}(\vartheta)\right)-U_{N}(\vartheta)+U_{N-2}(\vartheta)=0 .
$$

Using the Chebyshev polynomials $T_{n}$ of first kind, (48) can be also expressed as $\cos \xi T_{N+1}(\vartheta)-T_{N}(\vartheta)=0, \vartheta=$ $\cos \eta_{\kappa}$. The eigenvectors are given by a nontrivial constant multiple of the vector with components (41) or, in simpler form analogous to (47), for $\kappa \in \mathbb{Z}_{1}^{N}$,

$$
\begin{aligned}
a_{(\kappa) N+1+v} & =C_{N} \cos v \eta_{\kappa}, a_{(\kappa) v+1}=a_{(\kappa) 2 N+1-v}, v \in \mathbb{Z}_{0}^{N}, \quad \text { where } \\
C_{N}^{-2} & =N+\frac{1}{2}+\frac{1}{2}\left(\vartheta^{2}-1\right) \frac{\sin ^{2} \xi}{(\vartheta \cos \xi-1)^{2}} U_{N-1}^{2}(\vartheta) .
\end{aligned}
$$

The normalization represented by the symbol $C_{N}$ in (51) is derived in Appendix 2.2.

4.2c Surface modes: It is well known that the surface wave may exist below the pass band envelope for these lattice models with nearest-neighbour interactions in the presence of perturbations $[12,54]$. Indeed, in the case of $\mathfrak{I}_{\circ}^{\circ}$, a critical value of $\xi$, a 'threshold' in the surface wave dispersion relation, is given by $\vartheta=\cos \eta_{\kappa}= \pm 1$. Recall the wellknown identity (102b) ${ }_{3}$ [58]; its substitution in (39) implies that either $\cos \xi=1$ or $\cos \xi=\frac{N-1}{N+1}$, andeither $\cos \xi=-1$ or $\cos \xi=-\frac{N-1}{N+1}$. Note that both such critical points $\xi$ approach either 0 or $\pi$ separately, as $N \rightarrow \infty$ (implying a surface wave dispersion relation for the semi-infinite case). Simplifying the corresponding value $\Omega_{\kappa}^{2}$ obtained from (37), it is found to be equal to $\frac{2}{3}\left(12-((3 N+2) /(N+1))^{2}\right)$. Let

$$
\xi_{s}=\cos ^{-1} \frac{N-1}{N+1} \in[0, \pi] .
$$

Then one of the surface modes exists for $\xi \in\left(\xi_{s}, \pi\right] \cup$ $\left[-\pi,-\xi_{s}\right)$ while another surface mode exists for $\xi \in\left(\xi_{s}-\pi, \pi-\xi_{s}\right)$. For the odd modes in case of $\mathfrak{I}_{\circ}^{\circ}$ with $N=2 N$, using the identities $(2.29,2.30)$ in [58], it is found that $W_{N}(+1)=(2 N+1), W_{N}(-1)=(-1)^{N}$; again it is easy to see that either $\cos \xi=(2 N-1) /(2 N+1)$ or $\cos \xi=-1$, so that one of the surface modes is odd while the other is even. See the region between abruptly ending dispersion curves on the contour in figure 5 (left) and corresponding dashed portions in dispersion curves in figure $4 \mathrm{~b}$. Notice that the surface mode dispersion curves in figure $4 \mathrm{~b}$ lie entirely in the stop band, as expected [12, 54].

\subsection{Fixed-free boundary $\mathfrak{I}^{\bullet}$}

Consider $\mathfrak{I}_{\circ}^{\bullet}$ as schematically illustrated in the right side of figure 2. At $y=N$, the boundary condition is described by (33a), while at the lattice row $y=1,(34 b)$ holds. For the determination of the propagating waves, based on an equation analogous to (35), it is found that (10) holds for the normal modes, where $\mathbf{A}_{N}$ has the form (9) with definitions provided in (36) and $\delta_{N E}=\delta_{S W}=0, \delta_{N W}=$ $0, \delta_{S E}=-2$. Applying the results of [61], i.e., using (13), in this case for $\kappa \in \mathbb{Z}_{1}^{N}, \eta_{\kappa}$ satisfies $\cos \xi \sin (N+1) \eta_{\kappa}-$ $\sin N \eta_{\kappa}=0$. Using the Chebyshev polynomials $U_{n}$ of second kind, the condition is re-written as

$$
\cos \xi U_{N}(\vartheta)-U_{N-1}(\vartheta)=0 .
$$

Similar to the case of $\mathfrak{I}_{\circ}^{\circ}$, (53) also does not allow the determination of $\vartheta(\xi)$ or $\eta_{\kappa}(\xi)$ in a closed form [62]. After the value of $\eta_{\kappa}$ is obtained numerically that satisfies (53) for given $\xi$, an eigenvalue $\Lambda$ of $\mathbf{A}_{N}$ is obtained by (12). The dispersion relations are determined with $\Lambda=\frac{3}{2} \Omega_{\kappa}^{2}(\xi)=$ $\frac{3}{2} \Omega_{\mathfrak{z}}^{2}\left(\xi, \eta_{\kappa}\right)$, which are illustrated by figure $4 \mathrm{c}$, along with figure 5 (right). The index $\kappa$ is placed in such a way that $\Omega_{\kappa}(0)$ is an increasing sequence with respect to $\kappa$. The eigenvectors may be found in the same manner as before. In particular, the eigenvector is a constant multiple of the 
vector with components $\sin v \eta_{\kappa}$. Normalizing the same (as shown in the Appendix 2.2) it is found that, for $\kappa \in \mathbb{Z}_{1}^{N}$,

$a_{(\kappa) v}=C_{N} \sin v \eta_{\kappa}, v \in \mathbb{Z}_{1}^{N}$, where $C_{N}^{-2}=\frac{1}{4}\left(2 N+1-\sin ^{2} \xi U_{N}^{2}(\vartheta)\right)$.

Instead of solving for $\eta_{\kappa}$, alternatively, $\Omega_{\kappa}^{2}$ can be determined directly such that (53) holds. In particular, analogous to (42), the function $\omega^{2}=\Omega_{\kappa}^{2}(\xi)$ is implicitly given by $\frac{1}{2} \prod_{j=1}^{N}\left(6-2 \cos 2 \xi-\frac{3}{2} \Omega_{\kappa}^{2}-4 \cos \xi \cos \frac{j}{N+1} \pi\right)-\prod_{j=1}^{N-1}(6-$ $\left.2 \cos 2 \xi-\frac{3}{2} \Omega_{\kappa}^{2}-4 \cos \xi \cos \frac{j}{N} \pi\right)=0$. Using the same equation (44), when $\cos \xi \neq 0$ (i.e. $\xi \neq \pm \frac{1}{2} \pi$ ), $\eta_{\kappa}$ can be obtained from $\cos \eta_{\kappa}(\xi)=\left(6-2 \cos 2 \xi-\frac{3}{2} \Omega_{\kappa}^{2}(\xi)\right) /$ $(4 \cos \xi)$. Indeed, $\eta_{\kappa}$ is real provided it does not correspond to a surface mode (localized wave along $y$ ). For $\xi= \pm \frac{1}{2}$, (53) can be used directly to calculate $\eta_{\kappa}$. Also, note that an application of the second method, as described in section 4.2 , yields the same expressions.

4.3a Surface modes: A surface wave exists below the pass band envelope (illustrated by grey-coloured shaded region in figure 4(c)). Clearly, a critical value of $\xi$ that signals the threshold for surface wave dispersion relation satisfies $\cos \eta_{\kappa}(\xi)= \pm 1 . \quad$ Using $\quad(102 \mathrm{~b})_{3}, \quad$ either $\cos \xi=$ $N /(N+1)$ or $\cos \xi=-N /(N+1)$. Analogous to the case of $\mathfrak{I}_{\circ}^{\circ}$, both critical values of $\xi$ approach $0, \pi$ as $N \rightarrow \infty$. The values of the frequency $\omega$ corresponding to the endpoints of the surface wave dispersion curves are easy to find as $\frac{2}{3}\left(12-((3 N+2)(N+1))^{2}\right)$. Let

$$
\xi_{s}=\cos ^{-1} \frac{N}{N+1} \in[0, \pi] .
$$

Then only one surface mode exists for fixed-free boundary when $\xi \in\left(\xi_{s}, \pi-\xi_{s}\right) \cup\left(-\pi+\xi_{s},-\xi_{s}\right)$. See the region between abruptly ending dispersion curves on the contour in figure 5 (right) and corresponding dashed portions in dispersion curves in figure $4 \mathrm{c}$. Notice that the surface mode dispersion curves in figure $4 \mathrm{c}$ lie entirely in the stop band as expected $[12,54]$.

\subsection{Periodic boundary $\mathfrak{I} \odot$}

At the upper boundary row of $\mathfrak{I} \odot$ (see the illustration in figure 2), i.e., $y=N$, the condition (33c) holds, while for $y=1$, (34c) holds. Hence, based on an equation analogous to (35), (10) holds for the normal modes, where $\mathbf{A}_{N}$ has the form (9) with definitions provided in (36) and $\delta_{N E}=\delta_{S W}=\Upsilon, \delta_{N W}=\delta_{S E}=0 . \quad$ In particular, it is observed that $\mathbf{A}_{N}$ is a circulant matrix; consequently, the eigenpairs are straightforward to find as (30). An alternative derivation of this case is identical to that for the square lattice (except for replacing the expression of $\lambda$ by that provided for triangular lattice); hence it is not repeated here along with (31) provided in section 3.4. The expression (31) has been plotted and shown in figure $4 \mathrm{~d}$ marking the doubly repeated dispersion relations (except for the uppermost and lowermost). An application of the second method, as described in section 4.2, yields the same expressions. Notice that the periodic boundary condition for $\mathfrak{I}_{N}$ is possible only when $N$ is an even number (see Remark 10). For $N=2 N$, a possible case to investigate is that of 'odd' and 'even' modes with respect to the rectangular lattice strip $\mathfrak{R}_{N}$ (containing $\mathfrak{I}_{N}$ ); in this context, see also Remark 10.

4.4a 'Odd' modes: For odd modes, (10) holds where $\mathbf{A}_{N}$ has the form (9) with definitions provided in (36) and $\delta_{N E}=$ $\delta_{S W}=0, \delta_{N W}=-\Upsilon, \delta_{S E}=-\Upsilon$. This results in the condition $\Upsilon^{2} \sin (N+1) \eta_{\kappa}+\Upsilon^{2} \sin (N-1) \eta_{\kappa}+2 \Upsilon^{2} \sin N \eta_{\kappa}=$ 0 , which is, in terms of the Chebyshev polynomials of second kind,

$$
(\vartheta+1) U_{N-1}(\vartheta)=0, \vartheta=\cos \eta_{\kappa} .
$$

Hence, $\eta_{\kappa}=\kappa \pi / N, \kappa$ lies in $\mathbb{Z}_{1}^{N}$ and $a_{(\kappa) N+v}=\frac{1}{\sqrt{N}} \sin$ $\left(v-\frac{1}{2}\right) \eta_{\kappa}, v \in \mathbb{Z}_{1}^{N}$.

$4.4 \mathrm{~b}$ 'Even' modes: In a manner similar to the odd modes, consider the even modes. Hence (10) holds, where $\mathbf{A}_{N}$ has the form (9) with definitions provided in (36) and $\delta_{N E}=$ $\delta_{S W}=0, \delta_{N W}=\Upsilon, \delta_{S E}=\Upsilon$. This results in the condition $\Upsilon^{2} \sin (N+1) \eta_{\kappa}+\Upsilon^{2} \sin (N-1) \eta_{\kappa}-2 \Upsilon^{2} \sin N \eta_{\kappa}=0$.

Using the Chebyshev polynomials of second kind,

$$
(\vartheta-1) U_{N-1}(\vartheta)=0, \vartheta=\cos \eta_{\kappa} .
$$

Hence, $\quad \eta_{\kappa}=\kappa \pi / N, \quad \kappa$ lies in $\mathbb{Z}_{0}^{N-1}$ and $a_{(\kappa) N+v}=$ $\left(\left(\frac{1}{\sqrt{2}}-1\right) \delta_{\kappa, 0}+1\right) \frac{1}{\sqrt{N}} \cos \left(v-\frac{1}{2}\right) \eta_{\kappa}, v \in \mathbb{Z}_{1}^{N}$.

Remark 9 The reasoning analogous to Remark 8 holds for the periodic boundary condition also. Note that Remark 4 holds for the normal modes (including the surface modes wherever they exist) of all triangular lattice waveguides stated in table 2 .

Remark 10 The case $N=2 N+1$ for periodic boundary condition on $\mathfrak{R}_{N}$ can be interpreted as corresponding to a 'doubly' rolled triangular lattice strip; but this appears to be less relevant. Appendix 2.2 contains a detailed proof of the normalization that appears earlier, for example in (38), (41) and (54).

\section{Square lattice model $\hat{\mathfrak{S}}_{N}$ with next-nearest- neighbour interactions}

As an indication of the generalization of the analysis, presented so far in this paper, to the case of more 

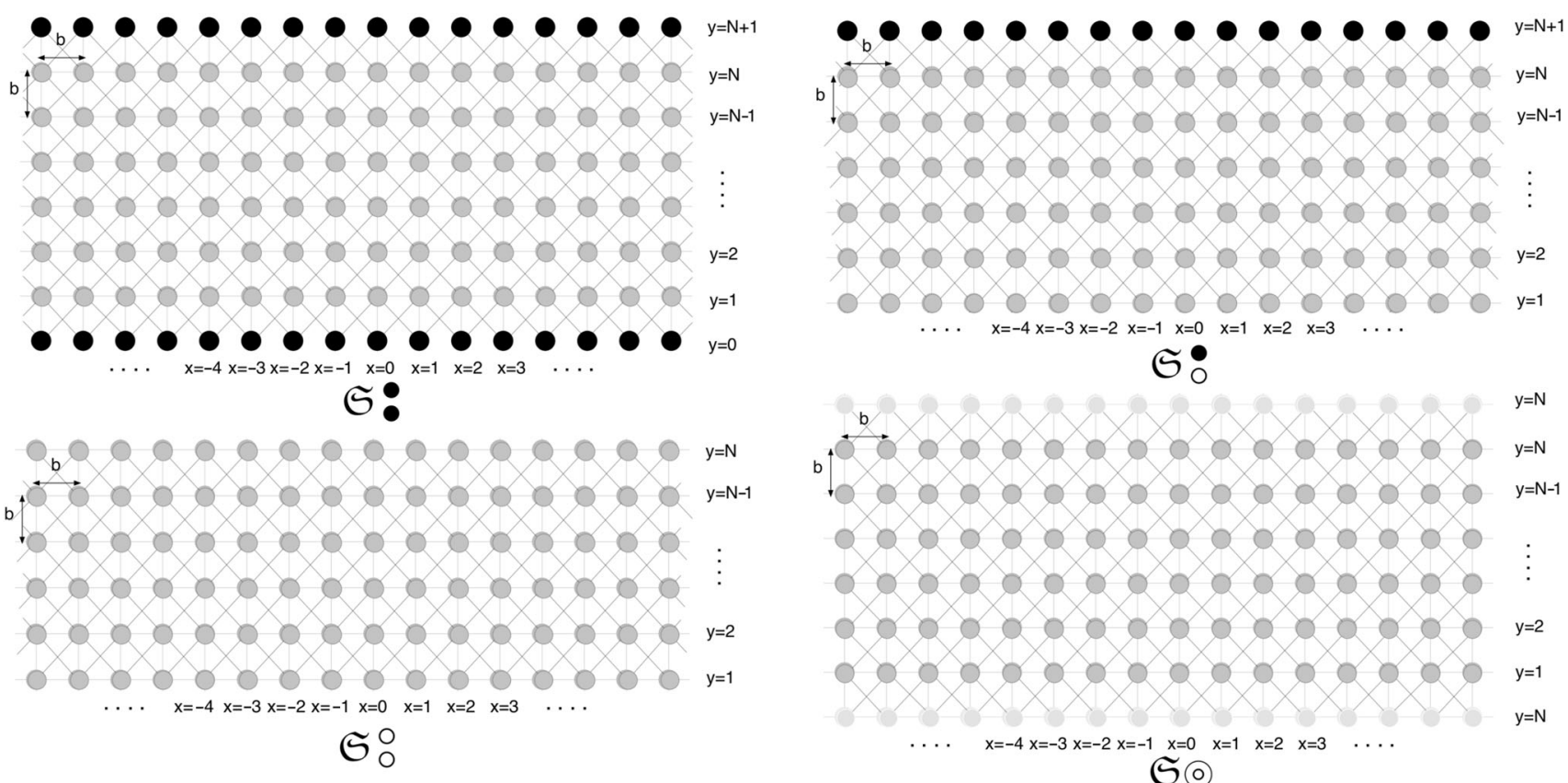

Figure 6. Illustration of the square lattice waveguides $\hat{\Xi}_{N}$ (with NNN interactions) corresponding to figure 2.

complicated interactions [12, 54], the square lattice of section 3 is extended to include the effect of four nextnearest-neighbour interactions (schematically shown in figure 6). The equation of motion for a particle in $\hat{\Xi}_{N}$ located at lattice site $(x, y)$, such that it does not belong to either of the strip boundary rows, is

$$
\begin{aligned}
M \frac{d^{2}}{d t^{2}} u_{x, y} & =K \sum_{i \in\{-1,1\}}\left(\left(u_{x+i, y}+u_{x, y+i}\right)+\gamma\left(u_{x+i, y+1}\right.\right. \\
& \left.\left.+u_{x+i, y-1}\right)\right)-4 K(1+\gamma) u_{x, y}, \quad \forall x \in \mathbb{Z}, y \in \mathbb{Z}_{2}^{N-1},
\end{aligned}
$$

where $\gamma \in \mathbb{R}$ is the ratio of the next-nearest-neighbour force constant $\hat{K}$ to the nearest-neighbor force constant $K$ (i.e. $\hat{K}=\gamma K)^{3}$. In the context of Remark 2, it is a straightforward exercise to obtain the traditional continuum model as a continuum limit of the square lattice model with nextnearest-neighbour interactions; in particular, it is easy to see that the shear wave speed $\left(c_{s}\right)$ is $\sqrt{1+2 \gamma} \sqrt{K b^{2} / M}$. Hence, it is assumed that $\gamma>-1 / 2$. For convenience of notation, let

$$
\Gamma_{0}:=1+2 \gamma .
$$

Let $M=\rho b^{3}, K=\Gamma_{0}^{-1} \mu b, t=t c_{s}$. The square lattice dispersion relation is $\omega^{2}=\frac{1}{b^{2}} \Omega_{\hat{\mathcal{S}}}^{2}(\xi, \eta)$, where

$$
\begin{aligned}
\Omega_{\hat{\Theta}}^{2}(\xi, \eta)= & \Gamma_{0}^{-1}\left(4 \sin ^{2} \frac{1}{2} \xi+4 \sin ^{2} \frac{1}{2} \eta+4 \gamma\left(\sin ^{2} \frac{1}{2}(\xi+\eta)\right.\right. \\
& \left.\left.+\sin ^{2} \frac{1}{2}(\xi-\eta)\right)\right), \quad(\xi, \eta) \in[-\pi, \pi]^{2} .
\end{aligned}
$$

Corresponding to the lattice waveguides listed in table 1, the boundary conditions at upper and lower rows studied in this paper are $\forall x \in \mathbb{Z}$, as followes: at $y=N$

$$
\begin{gathered}
-\Gamma_{0} \omega^{2} u_{x, y}=u_{x+1, y}+u_{x-1, y}+u_{x, y-1} \\
+\gamma\left(u_{x+1, y-1}+u_{x-1, y-1}\right)-4(1+\gamma) u_{x, y} \\
-\Gamma_{0} \omega^{2} u_{x, y}=u_{x+1, y}+u_{x-1, y}+u_{x, y-1} \\
+\gamma\left(u_{x+1, y-1}+u_{x-1, y-1}\right)-(3+2 \gamma) u_{x, y} \\
-\Gamma_{0} \omega^{2} u_{x, y}=u_{x+1, y}+u_{x-1, y}+u_{x, y-1}+u_{x, 1} \\
+\gamma\left(u_{x+1, y-1}+u_{x-1, y-1}\right)+\gamma\left(u_{x+1,1}+u_{x-1,1}\right) \\
-4(1+\gamma) u_{x, y} \quad(\odot),
\end{gathered}
$$

and at $y=1$

$$
\begin{aligned}
-\Gamma_{0} \omega^{2} u_{x, y}= & u_{x+1, y}+u_{x-1, y}+u_{x, y+1} \\
& +\gamma\left(u_{x+1, y+1}+u_{x-1, y+1}\right)-4(1+\gamma) u_{x, y}
\end{aligned}
$$

\footnotetext{
3 It is well known that the sign of $\gamma$ depends on the structural details for a specific material $[12,29,54,83,84]$.
} 


$$
\begin{gathered}
-\Gamma_{0} \omega^{2} u_{x, y}=u_{x+1, y}+u_{x-1, y}+u_{x, y+1} \\
+\gamma\left(u_{x+1, y+1}+u_{x-1, y+1}\right)-(3+2 \gamma) u_{x, y} \\
-\Gamma_{0} \omega^{2} u_{x, y}=u_{x+1, y}+u_{x-1, y}+u_{x, y+1}+u_{x, N} \\
+\gamma\left(u_{x+1, y-1}+u_{x-1, y-1}\right)+\gamma\left(u_{x+1, N}+u_{x-1, N}\right) \\
-4(1+\gamma) u_{x, y} \quad(\odot) .
\end{gathered}
$$

Clearly, $\gamma=0$ corresponds to the model without the nextnearest neighbour interactions as analysed in section 3 .

\subsection{Fixed boundary $\hat{\Theta}^{\bullet}$}

For the upper and lower boundary rows the conditions (61a) and (62a) hold, respectively. With the ansatz $u_{x, y}=a_{y} e^{i \kappa_{x} x}$, it follows that

$$
\begin{aligned}
-\Gamma_{0} \omega^{2} a_{y}= & (1+2 \gamma \cos \xi)\left(\left(1-\delta_{y, N}\right) a_{y+1}\right. \\
& \left.+\left(1-\delta_{y, 1}\right) a_{y-1}\right)+2 \cos \xi a_{y} \\
& -4(1+\gamma) a_{y}, y \in \mathbb{Z}_{1}^{N} .
\end{aligned}
$$

It is easy to see that $u$ is a propagating wave in $\hat{\Theta}_{\bullet}^{\bullet}$, with $\mathbf{A}_{N}$ of the form (9), if $a \in \mathbb{C}^{N}$ satisfies (10), where

$$
\Lambda=\Gamma_{0} \omega^{2}, \chi(\xi)=4(1+\gamma)-2 \cos \xi, \Upsilon=-1-2 \gamma \cos \xi
$$

and $\delta_{N E}=\delta_{S W}=0, \delta_{N W}=\delta_{S E}=0$. For convenience of notation, let

$$
\Gamma(\xi):=1+2 \gamma \cos \xi
$$

so that $\Upsilon=-\Gamma$ (as defined in (64)). Notice that $\Gamma(0)=1+2 \gamma=\Gamma_{0}$. According to [61], the eigenvalue problem (10) is solved by the eigenpairs described therein; in particular, each eigenvalue $\Lambda$ is described by the general form (12), where $\eta_{\kappa}$ are determined by a specific condition depending on $\mathbf{A}_{N}$ (13). Thus, the dispersion relations and normal modes are given by

$$
\begin{aligned}
& \omega^{2}=\Omega_{\kappa}^{2}(\xi)=\Gamma_{0}^{-1}\left(4 \sin ^{2} \frac{1}{2} \eta_{\kappa}+4 \sin ^{2} \frac{1}{2} \xi+4 \gamma\left(\sin ^{2} \frac{1}{2}\left(\xi+\eta_{\kappa}\right)\right.\right. \\
& \left.\left.+\sin ^{2} \frac{1}{2}\left(\xi-\eta_{\kappa}\right)\right)\right)=\Omega_{\hat{\Theta}}^{2}\left(\xi, \eta_{\kappa}\right), \\
& a_{(\kappa) v}=\sqrt{\frac{2}{N+1}} \sin v \eta_{\kappa}, v \in \mathbb{Z}_{1}^{N}, \quad \eta_{\kappa}=\frac{\kappa \pi}{N+1}, \kappa \in \mathbb{Z}_{1}^{N} .
\end{aligned}
$$

The expression (66) for the dispersion relations has been plotted and shown in figure 7a. The odd modes correspond to even values of $\kappa$, while the even modes correspond to odd values of $\kappa$. Similar to the case of triangular lattice $\mathfrak{I}^{\bullet}$ presented in section 4.1, the detailed reasoning for $\hat{\mathfrak{G}}_{\mathbf{0}}^{\bullet}$ is also the same as that for the square lattice waveguide $\mathfrak{S}^{\bullet}$ : (provided in section 3.1).

Remark $11 \quad$ Let $\xi_{*} \in[0, \pi]$ such that $\Gamma\left(\xi_{*}\right)=1+$ $2 \gamma \cos \xi_{*}=0$; clearly for the existence of $\xi_{*}$, it is required that $\gamma>1 / 2$ (since $\gamma>-1 / 2$ ). When the latter holds, it is evident from the four choices of $\gamma$ in figure 7 that the character of the pass band for $\hat{\mathfrak{S}}_{N}$ changes and resembles more of that for the triangular lattice. Note that when $\gamma>\frac{1}{2}$, it is possible that there exists a flat band (zero group velocity); in general, there is a possibility of a flat band whenever $\cos \eta_{\kappa}=-\frac{1}{2 \gamma}$ for $\gamma>\frac{1}{2}$, i.e., $\frac{N+1}{\pi} \arccos \left(-\frac{1}{2 \gamma}\right)$ is an integer.

\subsection{Free boundary $\hat{\mathfrak{\Xi}}_{0}^{\circ}$}

For $\hat{\Theta}_{\circ}^{\circ}$ (figure 6), i.e., $\hat{\Theta}_{N}$ with a free upper and lower boundary, Eqs. (61b) and (62b) hold, respectively. Again, based on an equation analogous to (63), it is found that (10) holds for the normal modes, where $\mathbf{A}_{N}$ has the form (9) with (64) and $\delta_{N E}=\delta_{S W}=0, \delta_{N W}=\delta_{S E}=-1-2 \gamma$. In this context, it is also useful to recollect the analysis of $\mathfrak{I}^{\circ}$ in section 4.2. Using (13), it is found that the dispersion relations are given by (66b), where $\eta_{\kappa}$ is such that for $\kappa \in \mathbb{Z}_{1}^{N}, \Gamma(\xi)^{2} \sin (N+1) \eta_{\kappa}+\Gamma_{0}^{2} \sin (N-1) \eta_{\kappa}-2 \Gamma_{0} \Gamma(\xi)$ $\sin N \eta_{\kappa}=0$. Indeed, using the Chebyshev polynomials $U_{n}$ of second kind, the latter is re-written as

$$
\Gamma(\xi)^{2} U_{N}(\vartheta)+\Gamma_{0}^{2} U_{N-2}(\vartheta)-2 \Gamma_{0} \Gamma(\xi) U_{N-1}(\vartheta)=0 .
$$

Using (66b), it is clear that $\eta_{\kappa}$ is a function of $\xi$ such that

$$
\cos \eta_{\kappa}=\vartheta=\frac{1}{2} \frac{4(1+\gamma)-2 \cos \xi-\Gamma_{0} \omega^{2}}{\Gamma(\xi)} .
$$

As remarked before, $\eta_{\kappa}(\xi)$ in (68) is real provided it does not correspond to a surface mode (discussed separately later). Also, it is assumed that $\Gamma(\xi) \neq 0$, while in the special case of $\Gamma(\xi)=0,(67)$ can be used directly to calculate $\eta_{\kappa}$. Once $\eta_{\kappa}$ is determined (numerically in this case) that satisfies Eq. (67) for given $\xi$ and $\kappa \in \mathbb{Z}_{1}^{N}$, the eigenvalue $\Lambda$ of $\mathbf{A}_{N}$ is given by (12) (and (64)) and thus the dispersion relations are determined with $\Lambda=\Omega_{\kappa}^{2}(\xi)=\Omega_{\hat{\Theta}}^{2}\left(\xi, \eta_{\kappa}\right)$. Applying the results of [61], the eigenvectors may be found for $\kappa \in \mathbb{Z}_{1}^{N}$, as a nontrivial constant multiple of the vector with components $-\Gamma(\xi) \sin v \eta_{\kappa}+\Gamma_{0} \sin (v-1) \eta_{\kappa}$; the same can be normalized to obtain, for $\kappa \in \mathbb{Z}_{1}^{N}$,

$$
a_{(\kappa) v}=\frac{-2 \Gamma(\xi) \sin v \eta_{\kappa}+2 \Gamma_{0} \sin (v-1) \eta_{\kappa}}{\sqrt{2 N\left(1+\Gamma(\xi)^{2}-2 \vartheta \Gamma_{0} \Gamma(\xi)\right)-2+2 \Gamma(\xi)^{2}}}, v \in \mathbb{Z}_{1}^{N} .
$$




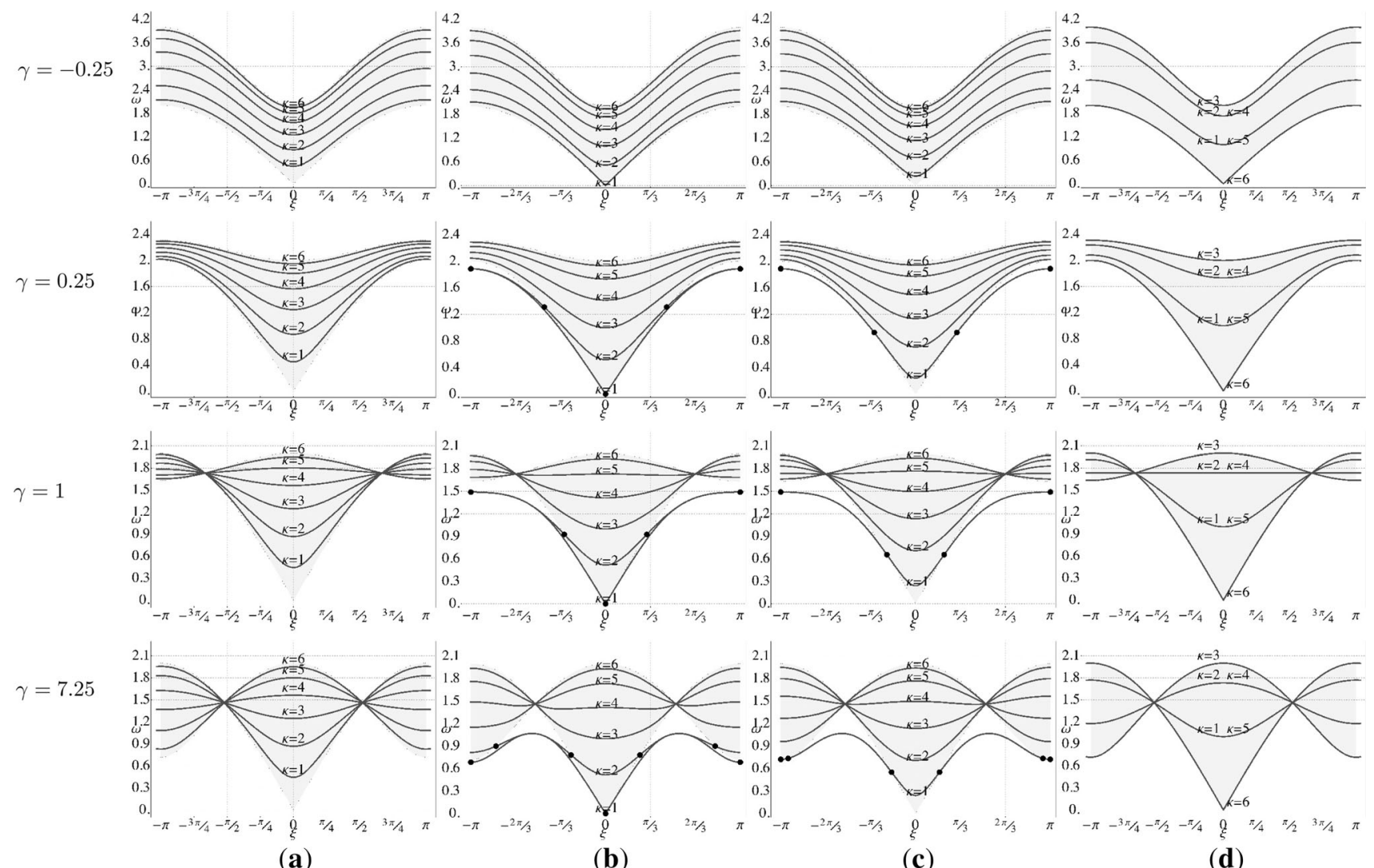

(a)

(b)

(c)

(d)

Figure 7. Dispersion relation $\Omega_{\kappa}, \kappa \in \mathbb{Z}_{1}^{N}$, with $N=6$ for $\hat{\Xi}_{N}$ with next-nearest-neighbour interactions (first row: $\gamma=-0.25$, second row: $\gamma=0.25$, third row: $\gamma=1$, fourth row: $\gamma=7.25$ ): (a) $\hat{\mathfrak{\Xi}}_{\bullet}^{\bullet}$, (b) $\hat{\mathfrak{\Xi}}_{\circ}^{\circ}$, (c) $\hat{\mathfrak{\Xi}}_{\bullet}^{\circ}$ and (d) $\hat{\mathfrak{\Xi}}_{\odot}$. The pass band envelope of the infinite lattice is illustrated by grey-coloured shaded region.

Instead of solving for $\eta_{\kappa}$, alternatively, $\Omega_{\kappa}^{2}$ can be solved for such that (67) holds. In particular, the function $\omega^{2}=\Omega_{\kappa}^{2}(\xi)$ is implicitly given by

$$
\begin{aligned}
& \prod_{j=1}^{N}\left(4(1+\gamma)-2 \cos \xi-\Omega_{\kappa}^{2}-\Gamma(\xi) \cos \frac{j \pi}{N+1}\right) \\
& +\Gamma_{0}^{2} \prod_{j=1}^{N-2}\left(4(1+\gamma)-2 \cos \xi-\Omega_{\kappa}^{2}-\Gamma(\xi) \cos \frac{j \pi}{N-1}\right) \\
& \quad-2 \Gamma_{0} \prod_{j=1}^{N-1}\left(4(1+\gamma)-2 \cos \xi-\Omega_{\kappa}^{2}-\Gamma(\xi) \cos \frac{j \pi}{N}\right)=0 .
\end{aligned}
$$

The index $\kappa$ is placed in such a way that $\Omega_{\kappa}(0)$ is an increasing sequence with respect to $\kappa$. figure $7 \mathrm{~b}$ and the left of figure 8 illustrate the dispersion curves for this case. Recall Remark 11 as well in the present context of the waveguide $\hat{\mathfrak{S}}_{\bullet}^{\bullet}$.

5.2a Odd modes: For $N=2 N$, by definition of an odd mode, it is required that $a_{v}=-a_{2 N+1-v}$, i.e., the problem is equivalent to finding the eigenvectors of (10) where $\mathbf{A}_{N}$ has the form (9) with definitions provided in (64) and $\delta_{N E}=$
$\delta_{S W}=0, \delta_{N W}=-1-2 \gamma, \delta_{S E}=\Gamma(\xi)$. Using (13), this leads to the condition

$$
\Gamma(\xi)\left(U_{N}(\vartheta)+U_{N-1}(\vartheta)\right)-\Gamma_{0}\left(U_{N-1}(\vartheta)+U_{N-2}(\vartheta)\right)=0 .
$$

The relation (71) can be also expressed in terms of the Chebyshev polynomials [58] of fourth kind $W$, leading to $\Gamma(\xi) W_{N}(\vartheta)-\Gamma_{0} W_{N-1}(\vartheta)=0, \vartheta=\cos \eta_{\kappa}$. The eigenvectors are given by (69) for suitable $\kappa$. An alternative and direct derivation of the eigenpairs is also possible using the second method. Using an equation analogous to (63), it is easy to see that the general solution $[65,82]$ for normal modes is given by (26b), where $\lambda$ satisfies

$$
\lambda+\lambda^{-1}=\mathscr{Q}(\xi):=\frac{4(1+\gamma)-2 \cos \xi-\Gamma_{0} \omega^{2}}{\Gamma(\xi)} .
$$

As in the case of triangular lattice waveguides, note that (21) also holds due to (72) and (68). Further, the expressions $\iota^{2}=\mathscr{Q}+2, h^{2}=2-2$ hold and $\lambda$ is given by (19). Due to the free boundary condition, it is required that $\Gamma_{0} C_{1} \lambda^{N}+\Gamma_{0} C_{2} \lambda^{-N}=\Gamma(\xi)\left(C_{1} \lambda^{N+1}+C_{2} \lambda^{-N-1}\right)$. Taking into account the symmetry, it is also required that $C_{1} \lambda+$ 

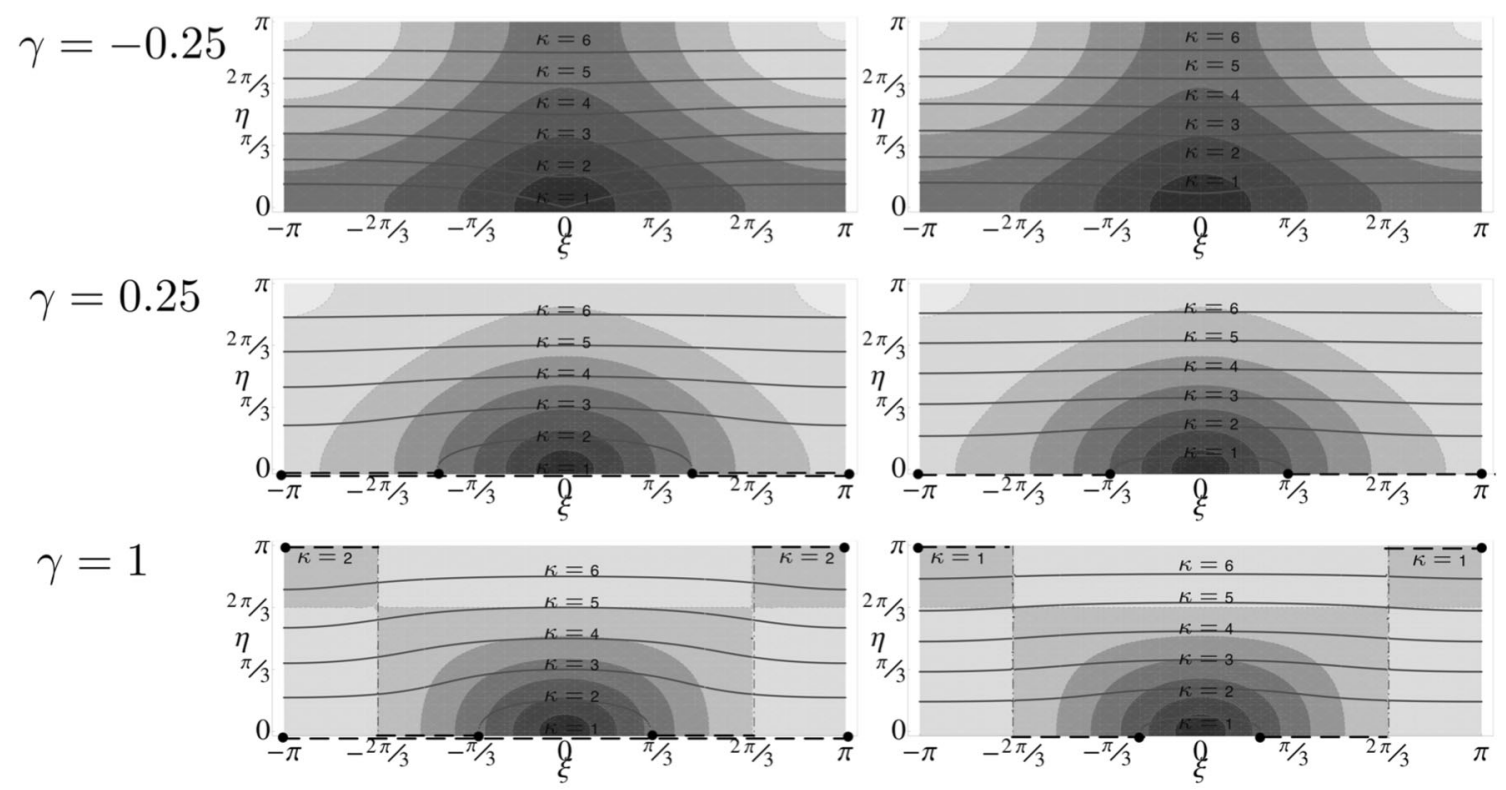

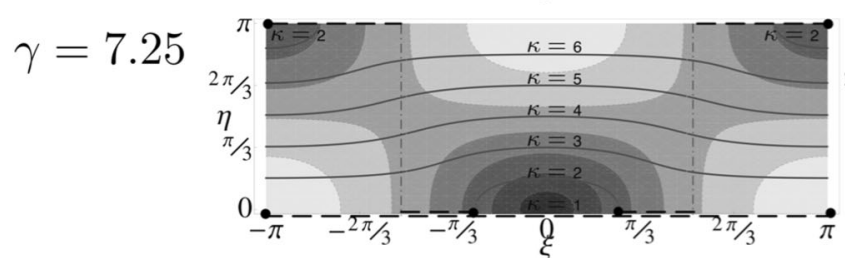

(b)

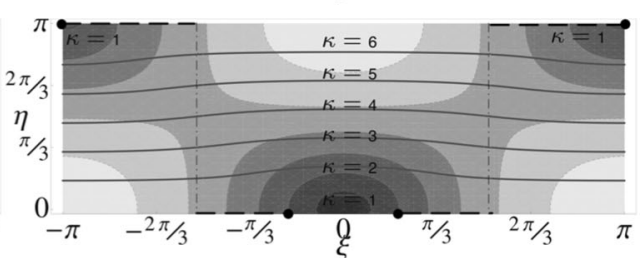

(c)

Figure 8. Contour plot of the square lattice dispersion relation $\Omega_{\hat{\Theta}}(\xi, \eta)$ and $\Omega_{\kappa}, \kappa \in \mathbb{Z}_{1}^{N}$ (first row: $\gamma=-0.25$, second row: $\gamma=0.25$, third row: $\gamma=1$, fourth row: $\gamma=7.25$ ), for $\hat{\mathfrak{S}}_{0}^{\circ}$ (left) and $\hat{\mathfrak{G}}_{\circ}^{\bullet}$ (right) with thick curves corresponding to the curves shown in figure $3 \mathrm{~b}$ and c, respectively. Notice the gaps (marked by dashed segments) due to the surface wave dispersion curves.

$C_{2} \lambda^{-1}=-C_{1}-C_{2}$. For a non-trivial solution $C_{1}, C_{2}$ of both equations, it is required that $\left(\Gamma_{0}-\lambda \Gamma(\xi)\right)(1+$ $\left.\lambda^{-1}\right) \lambda^{N}-\left(\Gamma_{0}-\lambda^{-1} \Gamma(\xi)\right)(1+\lambda) \lambda^{-N}=0$. Using the Chebyshev polynomials $U_{n}$ of second kind (after an application of (20)), it is easy to see that (71) follows, where $\vartheta$ is given by (21) and (72). The eigenvectors are also, consequently, described by the expression

$$
\begin{aligned}
a_{N+v} & =-a_{(\kappa) N-v+1} \\
& =\frac{\cos \left(v-\frac{1}{2}\right) \eta_{\kappa}}{\sqrt{N+(\vartheta+1) \gamma(1-\cos \xi)(1+\gamma(1+\cos \xi))^{-1} U_{N-1}^{2}(\vartheta)}}, v \in \mathbb{Z}_{1}^{N},
\end{aligned}
$$

where $\eta_{\kappa}, \vartheta$ satisfy (71). The normalization in (73) is derived in Appendix 2.2.

For $N=2 N+1$ and odd modes, it is required that $a_{v}=a_{2 N+2-v}$. Using the ansatz (16), the equations based on free-boundary condition and the symmetry admit a nontrivial $C_{1}, C_{2}$, provided $\left(\Gamma_{0}-\lambda \Gamma(\xi)\right)\left(1+\lambda^{-2}\right) \lambda^{N+1}-$ $\left(\Gamma_{0}-\lambda^{-1} \Gamma(\xi)\right)\left(1+\lambda^{2}\right) \lambda^{-N-1}=0$. After an application of (20) and (21), it follows that

$$
\Gamma(\xi) U_{N}(\vartheta)-\Gamma_{0} U_{N-1}(\vartheta)=0,
$$

where $\vartheta$ is given by (21). Referring to the expressions derived in section 5.3, (74) is same as that in the case of $\hat{\mathfrak{G}}^{\bullet}$ of width $N$; moreover, the same result is obtained using (10), where $\mathbf{A}_{N+1}$ has the form (9) with definitions provided in (36) and $\delta_{N E}=\delta_{S W}=0, \delta_{N W}=-1-2 \gamma, \delta_{S E}=0$. The eigenvectors corresponding to the dispersion relations, based on the value of $\eta_{\kappa}$ satisfying (74), are given by (69), or in specific form (see Appendix 2.2 for details concerning the normalization), for $\kappa \in \mathbb{Z}_{1}^{N}$,

$a_{(\kappa) N+1+v}=C_{N} \sin v \eta_{\kappa}, a_{(\kappa) v+1}=-a_{(\kappa) 2 N+1-v}, \quad v \in \mathbb{Z}_{0}^{N}$,

where $C_{N}^{-2}=N+\frac{1}{2}-2 \frac{\gamma(1-\cos \xi)(1+\gamma(1+\cos \xi))}{\Gamma_{0}^{2}} U_{N}^{2}(\vartheta)$.

5.2b Even modes: For even modes in $\hat{\mathfrak{G}}_{0}^{\circ}$ with $N=2 N$, it is required that $a_{v}=a_{2 N+1-v}$. The problem of finding propagating waves is equivalent to finding the eigenvectors of (10) where $\mathbf{A}_{N}$ has the form (9) and $\delta_{N E}=\delta_{S W}=0, \delta_{N W}=$ $-1-2 \gamma, \delta_{S E}=-1-2 \gamma \cos \xi$. After the application of (12) and (13), it is found that 
$\Gamma(\xi)\left(U_{N}(\vartheta)-U_{N-1}(\vartheta)\right)-\Gamma_{0}\left(U_{N-1}(\vartheta)-U_{N-2}(\vartheta)\right)=0$

Using the Chebyshev polynomials $V_{n}$ of third kind, the condition (77) can also be expressed as $\Gamma(\xi) V_{N}(\vartheta)-$ $\Gamma_{0} V_{N-1}(\vartheta)=0, \vartheta=\cos \eta_{\kappa}$. The eigenvectors are given by (69). An alternative derivation of the dispersion relation using the second method is included here as well. Using the definition of $\lambda$ in (20) and (72), the general solution for normal modes is given by (26b). For non-trivial $C_{1}, C_{2}$, in order to satisfy the free-boundary condition and the symmetry requirement, it is necessary that $\left(\Gamma_{0}-\lambda \Gamma(\xi)\right)(1-$ $\left.\lambda^{-1}\right) \lambda^{N}-\left(\Gamma_{0}-\lambda^{-1} \Gamma(\xi)\right)(1-\lambda) \lambda^{-N}=0$. using (20), it is found that (77) follows. With $\eta_{\kappa}$ that satisfies (77), the eigenvectors are described by the expression

$$
\begin{aligned}
a_{N+v} & =a_{(\kappa) N-v+1}=C_{N} \cos \left(v-\frac{1}{2}\right) \eta_{\kappa}, v \in \mathbb{Z}_{1}^{N}, \quad \text { where } \\
C_{N}^{-2} & =N+(\vartheta-1) \frac{1+\gamma(1+\cos \xi)}{\gamma(1-\cos \xi)} U_{N-1}^{2}(\vartheta) .
\end{aligned}
$$

See Appendix 2.3 for details concerning the normalization.

For even modes in $\hat{\mathfrak{\Xi}}_{0}^{\circ}$ with $N=2 N+1$, it is required that $a_{v}=a_{2 N+2-v}$. Using (16), the free-boundary condition and the symmetry, non-trivial $C_{1}, C_{2}$ exist provided $\left(\Gamma_{0}-\right.$ $\lambda \Gamma(\xi))\left(1-\lambda^{-2}\right) \lambda^{N+1}-\quad\left(\Gamma_{0}-\lambda^{-1} \Gamma(\xi)\right)\left(1-\lambda^{2}\right) \lambda^{-N-1}=$ 0 . Using the Chebyshev polynomials $U_{n}$ of second kind (20), it follows that

$$
\Gamma(\xi)\left(U_{N+1}(\vartheta)-U_{N-1}(\vartheta)\right)-\Gamma_{0}\left(U_{N}(\vartheta)-U_{N-2}(\vartheta)\right)=0 .
$$

In terms of the Chebyshev polynomials $T_{n}$ of the first kind, (79) becomes $\Gamma(\xi) T_{N+1}(\vartheta)-\Gamma_{0} T_{N}(\vartheta)=0, \vartheta=\cos \eta_{\kappa}$. The eigenvectors are given by (69) or, in simpler form analogous to (75), for $\kappa \in \mathbb{Z}_{1}^{N}$,

$$
a_{(\kappa) N+1+v}=C_{N} \cos v \eta_{\kappa}, a_{(\kappa) v+1}=a_{(\kappa) 2 N+1-v}, \quad v \in \mathbb{Z}_{0}^{N},
$$

where the normalization constant (Appendix 2.3) is

$$
C_{N}^{-2}=N+\frac{1}{2}+2 \gamma\left(\vartheta^{2}-1\right) \frac{1-\cos \xi+\gamma \sin ^{2} \xi}{(\vartheta-1+2 \gamma(\vartheta \cos \xi-1))^{2}} U_{N-1}^{2}(\vartheta)
$$

5.2c Surface modes: A critical value of $\xi$, a 'threshold' in the surface wave dispersion relation, is given by $\vartheta=$ $\cos \eta_{\kappa}= \pm 1$, assuming $\gamma \neq 0$. Recall the well-known identity [58] $U_{N}( \pm 1)=(N+1)(-1)^{\left(\frac{1}{2} \mp \frac{1}{2}\right) N}$ (included as $(102 \mathrm{~b})_{3}$ in Appendix 1); its substitution in (67) implies that (recall that $\Gamma_{0}>0$ )

$$
\alpha+\alpha^{-1} \mp 2+\left(\alpha-\alpha^{-1}\right) N^{-1}=0, \alpha=\Gamma(\xi) / \Gamma_{0} .
$$

Hence, either $\cos \xi=1$ or $\cos \xi=\frac{N-1-\gamma^{-1}}{N+1}$, andeither $\cos \xi$ $=-1-\gamma^{-1}$ or $\cos \xi=-\frac{N-1+N \gamma^{-1}}{N+1}$. Note that the first such critical point $\xi$ approaches 0 , as $N \rightarrow \infty$; however, the second critical point $\xi$ approaches $\cos ^{-1}\left(-1-\gamma^{-1}\right)$, as $N \rightarrow \infty$, but since $\Gamma_{0}>0$, this implies that there is only one surface wave dispersion relation for the semi-infinite case for $\xi \in[-\pi, \pi] \backslash\{0\}$. Let

$$
\begin{aligned}
\xi_{s 1} & =\cos ^{-1} \frac{N-1-\gamma^{-1}}{N+1} \in[0, \pi], \xi_{s 2} \\
& =\cos ^{-1}(-1) \frac{N-1+N \gamma^{-1}}{N+1} \in[0, \pi] .
\end{aligned}
$$

For real $\xi_{s 1}$, it is required that $-1<\left(N-1-\gamma^{-1}\right) /$ $(N+1)<1$, which can be reduced to the equivalent condition $\gamma>\frac{1}{2} N^{-1}=: \gamma_{*}$. Similarly, for real $\xi_{s 2}$, it is required that $-1<\left(N-1+N \gamma^{-1}\right) /(N+1)<1$ which also reduces to an equivalent condition $\gamma>\frac{1}{2} N=: \gamma^{*}$. Thus, as $N \rightarrow \infty$, and the surface wave dispersion relation for the semi-infinite case exists only for $\gamma>0$. Thus, $-\frac{1}{2}<\gamma \leq 0$ implies no such surface wave dispersion relation for the semi-infinite case as observed earlier for $\gamma=0$.

Assuming $\gamma_{*}<\gamma<\gamma^{*}$, a critical value of $\xi$, a 'threshold' in the surface wave dispersion relation is given by $\vartheta=$ $\cos \eta_{\kappa}=+1$, assuming $\gamma \neq 0$. Then one of the surface modes exists only for $\xi \in\left(\xi_{s 1}, \pi\right] \cup\left[-\pi,-\xi_{s 1}\right)$, while the other surface mode exists for all $\xi \in[-\pi, \pi] \backslash\{0\}$. In the alternate case, assuming $\gamma>\frac{1}{2} N$, there are two critical points (thresholds) as both $\xi_{s 1}, \xi_{s 2}$ are real. Thus, one of the surface modes exists for $\xi \in\left[-\xi_{s 2}, \xi_{s 2}\right]$ while another surface mode exists for $\xi \in\left[\xi_{s 1}, \pi\right] \cup\left[-\pi,-\xi_{s 1}\right]$. Analogous to the triangular lattice (section 4.2c), for the odd modes (71) in case of $\hat{\mathfrak{\Xi}}_{0}^{\circ}$ with $N=2 N$, it is found that $W_{N}(+1)=(2 N+1), W_{N}(-1)=(-1)^{N}$; again it is easy to see that either $\cos \xi=\left(2 N-1-\gamma^{-1}\right) /(2 N+1)$ or $\cos \xi=-1-\gamma^{-1}$, so that one of the two surface modes is odd while the other is even. Notice that the surface mode dispersion curves in figure $7 \mathrm{~b}$ lie entirely in the stop band $[12,54]$.

Remark 12 In the context of figure $7 \mathrm{~b}$, it is worth observing that the first choice $\gamma=-0.25>-\frac{1}{2}$, the second choice $\gamma=0.25 \in\left(\gamma_{*}, \gamma^{*}\right)$ but $\gamma<\frac{1}{2}$ (recall Remark 11), the third choice $\gamma=1 \in\left(\gamma_{*}, \gamma^{*}\right)$ but $\gamma>\frac{1}{2}$, and, finally, the fourth choice $\gamma=7.25>\gamma^{*}$. Note that for $N=$ $6, \gamma_{*} \approx 0.08$ and $\gamma^{*}=3$.

\subsection{Fixed-free boundary $\hat{\mathfrak{\Xi}}^{\bullet}$}

Consider $\hat{\mathfrak{G}}_{\circ}^{\bullet}$ as shown in figure 6 . The equation of motion for the lattice row at $y=1$ is (61a), while at $y=N$ it is (62b). Naturally, there is a close connection 
with the results established in section 4.3 for $\hat{\mathfrak{I}}_{0}$. It is found that (10) holds for the normal modes, where $\mathbf{A}_{N}$ has the form (9) with definitions provided in (64) and $\delta_{N E}=\delta_{S W}=0, \delta_{N W}=0, \delta_{S E}=-1-2 \gamma$. Applying the results of [61], i.e., using (13), in this case for $\kappa \in \mathbb{Z}_{1}^{N}, \eta_{\kappa}$ satisfies $\Gamma(\xi) \sin (N+1) \eta_{\kappa}-\Gamma_{0} \sin N \eta_{\kappa}=0$. Using the Chebyshev polynomials $U_{n}$ of second kind (20), the same condition is re-written as

$$
\Gamma(\xi) U_{N}(\vartheta)-\Gamma_{0} U_{N-1}(\vartheta)=0 .
$$

The dispersion relations are determined with $\Lambda=\Omega_{\kappa}^{2}(\xi)=$ $\Omega_{\hat{\S}}^{2}\left(\xi, \eta_{\kappa}\right)$, which are illustrated by figure $3 \mathrm{c}$, along with figure 8 (right). The index $\kappa$ is placed in such a way that $\Omega_{\kappa}(0)$ is an increasing sequence with respect to $\kappa$. Recall Remark 11 as well in the context of the appearance of a special point for $\xi$. As before, each eigenvector may be found in the same manner as before; in particular, it is a constant multiple of the vector with components $\sin v \eta_{\kappa}$. Normalizing the same (Appendix 2.2), it is found that for $\kappa \in \mathbb{Z}_{1}^{N}$,

$a_{(\kappa) v}=C_{N} \sin v \eta_{\kappa}, v \in \mathbb{Z}_{1}^{N}$, where

$C_{N}^{-2}=\frac{1}{2}\left(N+\frac{1}{2}-2 \frac{\gamma(1-\cos \xi)(1+\gamma(1+\cos \xi))}{\Gamma_{0}^{2}} U_{N}^{2}\right)$.

Analogous to (70), the function $\omega^{2}=\Omega_{\kappa}^{2}(\xi)$ is also implicitly given by

$$
\begin{aligned}
& \prod_{j=1}^{N}\left(4(1+\gamma)-2 \cos \xi-\Omega_{\kappa}^{2}-\Gamma(\xi) \cos \frac{j}{N+1} \pi\right) \\
& \quad-\Gamma_{0} \prod_{j=1}^{N-1}\left(4(1+\gamma)-2 \cos \xi-\Omega_{\kappa}^{2}-\Gamma(\xi) \cos \frac{j}{N} \pi\right)=0 .
\end{aligned}
$$

Using (72), when $\Gamma(\xi) \neq 0, \eta_{\kappa}$ can be obtained from $\cos \eta_{\kappa}(\xi)=\left(4(1+\gamma)-2 \cos \xi-\Gamma_{0} \omega^{2}\right) /(2 \Gamma(\xi))$. Indeed, $\eta_{\kappa}$ is real provided it does not correspond to a surface mode (localized wave along $y$ ). For $\Gamma(\xi)=0$, (84) can be used directly to calculate $\eta_{\kappa}$. An application of the second method, as described in section 5.2, yields the same expressions.

5.3a Surface modes: A surface wave exists below the pass band envelope (illustrated by grey-coloured shaded region in Figure 3(c)). Clearly, a critical value of $\xi$ that signals the threshold for surface wave dispersion relation satisfies $\cos \eta_{\kappa}(\xi)= \pm 1$. Using $\quad(102 \mathrm{~b})_{3}$, i.e. $\quad U_{N}( \pm 1)=$ $(N+1)(-1)^{\left(\frac{1}{2} \mp \frac{1}{2}\right) N}$, it is found that $\Gamma(\xi)(N+1)= \pm \Gamma_{0} N$, so that either $\cos \xi=\left(N-\frac{1}{2} \gamma^{-1}\right) /(N+1)$ or $\cos \xi=(-N-$ $\left.\left(\frac{1}{2}+N\right) \gamma^{-1}\right) /(N+1)$. Analogous to the case of $\hat{\mathfrak{\Xi}}_{0}^{\circ}$, both critical values of $\xi$ approach $0, \pi$, as $N \rightarrow \infty$. Let $\xi_{s 1}=\cos ^{-1} \frac{N-\frac{1}{2} \gamma^{-1}}{N+1} \in[0, \pi], \xi_{s 2}=\cos ^{-1} \frac{-N-\left(\frac{1}{2}+N\right) \gamma^{-1}}{N+1} \in[0, \pi]$.

For real $\xi_{s 1}$, it is required that $-1<\left(N-\frac{1}{2} \gamma^{-1}\right)(N+1)<1$, which can be reduced to the equivalent condition $\gamma>\frac{1}{2}(2 N+1)^{-1}=: \gamma_{* *}$. Similarly, for real $\xi_{s 2}$, it is required that $-1<\left(N+\left(\frac{1}{2}+N\right) \gamma^{-1}\right)(N+1)<1$, which also reduces to an equivalent condition $\gamma>\frac{1}{2}(2 N+1)=: \gamma^{* *}$. Thus, as $N \rightarrow \infty$, the surface wave dispersion relation for the semi-infinite case exists only for $\gamma>0$. The condition $-\frac{1}{2}<\gamma \leq 0$ implies no such surface wave dispersion relation for the semi-infinite case as observed earlier for $\gamma=0$.

Assuming that $\gamma$ satisfies $\gamma_{* *}<\gamma<\gamma^{* *}$, it is clear that a critical value of $\xi$ that signals the threshold for surface wave dispersion relation satisfies $\cos \eta_{\kappa}(\xi)=+1$. Then only one surface mode exists for fixed-free boundary when $\xi \in\left[-\pi,-\xi_{s}\right) \cup\left(\xi_{s}, \pi\right]$. Assuming that $\gamma>\gamma^{* *}$, there is only one surface wave mode and one part of its dispersion curve exists when $\xi \in\left[\xi_{s 1}, \xi_{s 2}\right] \cup\left[-\xi_{s 2},-\xi_{s 1}\right]$ with $\cos \eta_{\kappa}(\xi)=+1$, while another part exists when $\xi \in$ $\left[-\pi,-\xi_{s 2}\right] \cup\left[\xi_{s 2}, \pi\right]$ with $\cos \eta_{\kappa}(\xi)=-1$; see the region between abruptly ending dispersion curves on the contour in figure 8 (right) and corresponding dashed portions in dispersion curves in figure $7 \mathrm{c}$ for the latter. Notice that the surface mode dispersion curves in 7(c) lie entirely in the stop band as expected [12, 54].

Remark 13 Apart from the Remark 12 in the previous case, in the context of figure $7 \mathrm{c}$, it is worth observing that the second choice $\gamma=0.25 \in\left(\gamma_{* *}, \gamma^{* *}\right)$, the third choice $\gamma=1 \in\left(\gamma_{* *}, \gamma^{* *}\right)$ and, finally, the fourth choice $\gamma=7.25>\gamma^{* *}$; indeed, $\gamma_{* *} \approx 0.39$ and $\gamma^{* *}=6.5$ for $N=6$.

\subsection{Periodic boundary $\hat{\Xi} \odot$}

Due to the periodic boundary condition, (61d) and (62d) hold at upper and lower boundary rows, respectively. It follows that (10) holds, where $\mathbf{A}_{N}$ has the form (9) with (64) and $\delta_{N E}=\delta_{S W}=\Upsilon, \delta_{N W}=\delta_{S E}=0$; in particular, the eigenpairs are found to be the same as in (30). An alternative derivation of this case is identical to that for the square lattice with nearest-neighbour interactions only in section 3.4 (except for replacing the expression of $\lambda$ by that provided for the lattice model of this section). The expression (31) has been plotted and shown in figure $3 \mathrm{~d}$ marking the doubly repeated dispersion relations (except for the uppermost and lowermost). Recall Remark 11 as well in the context of $\gamma>\frac{1}{2}$. As a special case, following Remark 11, note that $\gamma=1$ is associated with a flat band; in general, there is a possibility of a flat band whenever $\cos \frac{2 \kappa \pi}{N}=-\frac{1}{2 \gamma}$ for $\gamma>\frac{1}{2}$, i.e., $\frac{N}{2 \pi} \arccos \left(-\frac{1}{2 \gamma}\right)$ is an integer. 
When $N=2 N$, the analysis and the resulting expressions for the odd modes and even modes are identical to those for the triangular lattice (sections $4.4 \mathrm{a}$ and $4.4 \mathrm{~b}$ ); the details are omitted. The case $N=2 N+1$ is considered later.

5.4a 'Odd' modes: For odd modes, (10) holds where $\mathbf{A}_{N}$ has the form (9) with definitions provided in (64) and $\delta_{N E}=$ $\delta_{S W}=0, \delta_{N W}=-\Upsilon, \delta_{S E}=0$. This results in the condition $\Upsilon^{2} \sin (N+1) \eta_{\kappa}+\Upsilon^{2} \sin N \eta_{\kappa}=0$, which is, in terms of the Chebyshev polynomials of second kind,

$$
U_{N}(\vartheta)+U_{N-1}(\vartheta)=0, \vartheta=\cos \eta_{\kappa} .
$$

The same relation, in terms of the Chebyshev polynomials of fourth kind, is $W_{N}(\vartheta)=0$. Hence, $\eta_{\kappa}=\kappa \pi /\left(N+\frac{1}{2}\right), \kappa$ lies in $\mathbb{Z}_{1}^{N}$ and $a_{(\kappa) N+1+v}=\sqrt{2 /(2 N+1)} \sin v \eta_{\kappa}, a_{(\kappa) v+1}=$ $-a_{(\kappa) 2 N+1-v}, v \in \mathbb{Z}_{0}^{N}$.

$5.4 \mathrm{~b}$ 'Even' modes: For even modes in $\hat{\Xi}_{\odot}$ with $N=2 N+1$, it is required that $a_{v}=a_{2 N+2-v}$. Using (16), due to the periodic boundary condition at $v=1$, and the symmetry at $v=N+1$, it is required that $C_{1} \lambda+C_{2} \lambda^{-1}=$ $C_{1}+C_{2}$ as well as $C_{1} \lambda^{N}+C_{2} \lambda^{-N}=C_{1} \lambda^{N+2}+C_{2} \lambda^{-N-2}$. Thus, non-trivial $C_{1}, C_{2}$ exist provided $(\lambda-1)\left(\lambda^{-N}-\right.$ $\left.\lambda^{-N-2}\right)-\left(\lambda^{-1}-1\right)\left(\lambda^{N}-\lambda^{N+2}\right)=0$. Equivalently, using the Chebyshev polynomials $U_{n}$ of second kind, the condition is $U_{N}(\vartheta)+U_{N-1}(\vartheta)-U_{N-2}(\vartheta)-U_{N+2}(\vartheta)=0$, i.e.,

$$
2(\vartheta-1)\left(U_{N}(\vartheta)+U_{N-1}(\vartheta)\right)=0,
$$

after an application of (102a) $)_{1}$. Using the Chebyshev polynomials $W_{n}$ of fourth kind, the dispersion relation can be also expressed as $(\vartheta-1) W_{N}(\vartheta)=0, \vartheta=\cos \eta_{\kappa}$. Hence, $\eta_{\kappa}=\kappa \pi /\left(N+\frac{1}{2}\right), \kappa$ lies in $\mathbb{Z}_{0}^{N}$ and the eigenvectors are given by $a_{(\kappa) N+1+v}=\left(\left(\frac{1}{\sqrt{2}}-1\right) \delta_{\kappa, 0}+1\right) \sqrt{2 /(2 N+1)}$ $\cos v \eta_{\kappa}, a_{(\kappa) v+1}=a_{(\kappa) 2 N+1-v}, v \in \mathbb{Z}_{0}^{N}$.

Remark 14 The reasoning analogous to Remark 8 holds for the periodic boundary condition on $\hat{\Xi}_{N}$ also. Note that Remark 4 holds for the normal modes (including the surface modes wherever they exist) of all square lattice waveguides stated in table 1.

\section{Discussion and related analysis}

\subsection{Even-odd factors of dispersion relations for symmetric boundary conditions}

In case the upper and lower boundary conditions are identical in the lattice waveguides, the forms of the appearance of the dispersion relations for even and odd modes, in each case studied earlier in the paper, are not unrelated. In fact, these results provide a factorization of the expression of the complete dispersion relation for the lattice waveguide, in terms of Chebyshev polynomials, as a product of two expressions, one of which corresponds to the even modes and other to the odd modes (similar to the identities stated as part of the expansion of the WienerHopf kernels encountered in [26]). In the following, the argument $\vartheta$ (21) (with (18), (44) and (68)) of the Chebyshev polynomials is suppressed, and $\eta$ is used in place of $\eta_{\kappa}$, for brevity.

1. $\mathfrak{G}_{:}^{\bullet}, \mathfrak{T}_{\bullet}^{\bullet}$ and $\hat{\mathfrak{S}}_{\bullet}^{\bullet}$ : using (22b) and (23b) for $N=2 N$,

$$
\left(U_{N}-U_{N-1}\right)\left(U_{N}+U_{N-1}\right)=V_{N} W_{N}=U_{2 N}=U_{N}(\text { by }(103 \mathrm{a})),
$$

while, using (22a) and (23a) for $N=2 N+1$,

$$
\left(U_{N+1}-U_{N-1}\right) U_{N}=2 T_{N+1} U_{N}=U_{2 N+1}=U_{N}(\text { by }(103 \mathrm{~b})),
$$

as expected with reference to (15).

2. $\mathfrak{S}_{\circ}^{\circ}$ : Indeed, using (26c) and (27a) for $N=2 N$,

$$
U_{N-1}\left(U_{N}-U_{N-2}\right) U_{N}=2 T_{N} U_{N-1}=U_{2 N-1}=U_{N-1}(\text { by }(103 \mathrm{~b})),
$$

while using (26a) and (27b) for $N=2 N+1$,

$$
\left(U_{N}+U_{N-1}\right)\left(U_{N}-U_{N-1}\right)=W_{N} V_{N}=U_{2 N}=U_{N-1}(\text { by }(103 \mathrm{a})),
$$

as expected with reference to (25).

3. $\mathfrak{T}_{0}^{\circ}$ : Using (48) and (43) for $N=2 N$,

$$
\begin{aligned}
& \left(\cos \xi V_{N}-V_{N-1}\right)\left(\cos \xi W_{N}-W_{N-1}\right) \\
& =\cos ^{2} \xi V_{N} W_{N}-\cos \xi\left(V_{N-1} W_{N}+V_{N} W_{N-1}\right)+V_{N-1} W_{N-1} \\
& =\cos ^{2} \xi U_{N}+U_{N-2}-2 \cos \xi U_{N-1}(\text { by }(103 \mathrm{a}) \text { and }(103 \mathrm{c})),
\end{aligned}
$$

while using (50) and (46) for $N=2 N+1$,

$$
\begin{aligned}
& 2\left(\cos \xi T_{N+1}-T_{N}\right)\left(\cos \xi U_{N}-U_{N-1}\right) \\
& =2 \cos ^{2} \xi T_{N+1} U_{N}-2 \cos \xi\left(T_{N} U_{N}+T_{N+1} U_{N-1}\right)+2 T_{N} U_{N-1} \\
& =\cos ^{2} \xi U_{N}+U_{N-2}-2 \cos \xi U_{N-1} \text { (by (103b) and (103d)), }
\end{aligned}
$$

as expected with reference to (39).

4. $\hat{\mathfrak{\Xi}}_{0}^{\circ}$ : Using (77) and (71) for $N=2 N$,

$$
\begin{aligned}
& \left(\Gamma(\xi) V_{N}-\Gamma_{0} V_{N-1}\right)\left(\Gamma(\xi) W_{N}-\Gamma_{0} W_{N-1}\right) \\
& =\Gamma(\xi)^{2} V_{N} W_{N}-\Gamma(\xi) \Gamma_{0}\left(V_{N-1} W_{N}+V_{N} W_{N-1}\right)+\Gamma_{0}^{2} V_{N-1} W_{N-1} \\
& =\Gamma(\xi)^{2} U_{N}+\Gamma_{0}^{2} U_{N-2}-2 \Gamma_{0} \Gamma(\xi) U_{N-1}(\text { by }(103 \mathrm{a}) \text { and }(103 \mathrm{c})),
\end{aligned}
$$

while using (79) and (74) for $N=2 N+1$, 


$$
\begin{aligned}
& 2\left(\Gamma(\xi) T_{N+1}-\Gamma_{0} T_{N}\right)\left(\Gamma(\xi) U_{N}-\Gamma_{0} U_{N-1}\right) \\
& =2 \Gamma(\xi)^{2} T_{N+1} U_{N}-2 \Gamma(\xi) \Gamma_{0}\left(T_{N} U_{N}+T_{N+1} U_{N-1}\right)+2 \Gamma_{0}^{2} T_{N} U_{N-1} \\
& =\Gamma(\xi)^{2} U_{N}+\Gamma_{0}^{2} U_{N-2}-2 \Gamma_{0} \Gamma(\xi) U_{N-1}(\text { by }(103 \mathrm{~b}) \text { and }(103 \mathrm{~d})),
\end{aligned}
$$

as expected with reference to (67).

5. $\odot \odot, ~ \mathfrak{I} \odot$ and $\hat{\mathfrak{\Xi}} \odot$ : Using (57) and (56) for $N=2 N$,

$$
\begin{aligned}
& 4\left(\vartheta^{2}-1\right) U_{N-1}^{2}=U_{2 N}-U_{2 N-2}-2 \\
& =U_{N}-U_{N-2}-2\left(\text { by }(103 \mathrm{e}) \text { and }(102 \mathrm{~b})_{1}\right),
\end{aligned}
$$

while using (89) and (88) for $N=2 N+1$,

$$
\begin{aligned}
& 2(\vartheta-1)\left(U_{N}+U_{N-1}\right)^{2}=U_{2 N+1}-U_{2 N-1}-2 \\
& =U_{N}-U_{N-2}-2\left(\mathrm{by}(103 \mathrm{f}) \text { and }(102 \mathrm{~b})_{1}\right),
\end{aligned}
$$

as expected with reference to (31).

\subsection{Features of $\hat{\mathfrak{\Xi}}_{N}$ and its relation with the first two models}

As discussed in the context of the square lattice model with next-nearest-neighbour interactions in section 5, there exist certain special values of the parameter $\gamma$. Summarizing these observations, the set of critical values of $\gamma$ are

1. $\gamma=-\frac{1}{2}: \gamma \leq-\frac{1}{2}$ leads to an unphysical situation when the shear wave speed is imaginary or zero;

2. $\gamma=\frac{1}{2}: \quad \gamma \geq \frac{1}{2}$ is associated with the appearance of a feature similar to that for the triangular lattice encountered before; in this context $\xi_{*}$ defined in Remark 11 can be inspected in figure 8 ;

3. $\gamma=\gamma_{* *}=\frac{1}{2}(2 N+1)^{-1}: \gamma>\gamma_{* *}$ is associated with the surface waves in the waveguide with fixed-free boundary conditions with dispersion curve of type shown in figure 7(c) for $\gamma=1$;

4. $\gamma=\gamma_{*}=\frac{1}{2} N^{-1}: \gamma>\gamma_{*}$ is associated with the surface waves in the waveguide with free boundary conditions with dispersion curve of type shown in figure $7 \mathrm{~b}$ for $\gamma=1$;

5. $\gamma=\gamma^{*}=\frac{1}{2} N: \gamma>\gamma^{*}$ is associated with the surface waves in the waveguide with free boundary conditions with dispersion curve of type shown in figure $7 \mathrm{~b}$ for $\gamma=7.25$

6. $\gamma=\gamma^{* *}=\frac{1}{2}(2 N+1): \gamma>\gamma^{* *}$ is associated with the surface waves in the waveguide with fixed-free boundary conditions with dispersion curve of type shown in figure $7(\mathrm{c})$ for $\gamma=7.25$.

As $\gamma \rightarrow 0$, the earlier results for the square lattice model (with nearest-neighbour interactions only) can be easily recovered. Moreover, and quite elegantly, for $\gamma \gg 1$, it is also easy to recognize the relevance of the results for the triangular lattice model (with nearest-neighbour interactions only). Thus, the square lattice model with nearest-neighbour and next-nearest-neighbour interactions can be interpreted as an intermediate case. A relook at the schematic in figures 2 and 6 for the square and triangular lattice models reveals the same immediately.

\subsection{Expressions for the dispersion relations}

Following the manipulations leading to the type of expressions (42), (70), etc, in order to obtain alternate expressions for the dispersion relations stated so far, consider the definition, for given integers $j$ and $n$ :

$$
\begin{aligned}
& F_{j, n}(\xi) \\
& =\left\{\begin{array}{cc}
4-2 \cos \xi-\omega^{2}-2 \cos \frac{j}{n+1} \pi & \text { for } \Xi_{\mathfrak{N}} \text { in section 2, } \\
6-2 \cos 2 \xi-\frac{3}{2} \omega^{2}-4 \cos \xi \cos \frac{j}{n+1} \pi & \text { for } \mathfrak{I}_{\mathfrak{N} \text { in section } 3,} \\
4(1+\gamma)-2 \cos \xi-\Gamma_{0} \omega^{2}-2 \Gamma(\xi) \cos \frac{j}{n+1} \pi & \text { for } \widehat{\Xi}_{\mathrm{N}} \text { in section } 4,
\end{array}\right.
\end{aligned}
$$

where $\kappa=1,2, \ldots, N$. Using the expansion of Chebyshev polynomials [56, 58], the dispersion relations for all the four cases (excluding the even-odd cases) can be simplified further; a summary of the resulting expressions is provided in table 3 .

\section{Concluding remarks}

The paper presents an analysis of the propagating modes of linear waves in several infinite lattice waveguides of square and triangular structure. The dispersion relations, the normal modes and detailed specifications of the surface waves are included. Some of the expressions stated in the paper are explicit, especially for the square lattice waveguides and most of the triangular lattice waveguides, while for some triangular lattice waveguides, these expressions are provided in terms of the roots of various kinds of linear combinations of the Chebyshev polynomials. The influence of next-nearestneighbour interactions is studied in the context of square lattice waveguides, where the corresponding mathematical formulation and analysis is found to be a natural extension of that for the square lattice and the triangular lattice with nearest-neighbour interactions. The techniques used in the paper can be applied and extended to other situations, such as bimaterial strips, etc, as well as for more realistic lattice models. It is possible to apply the results stated in this paper on a varied set of problems arising in diverse physical phenomena, including the current scientific and technological interests that involve the motion of crystalline solids at small scales. 
Table 3. Summary for $\mathfrak{\Xi}_{\mathfrak{N}}, \mathfrak{I}_{\mathfrak{N}}$ and $\hat{\mathfrak{\Xi}}_{N}$ (see also tables 1 and 2) using (100).

\begin{tabular}{|c|c|c|c|}
\hline S. no. & Strip & Dispersion relation & Modes \\
\hline 1 & $\mathrm{~s}^{*}$ & $\prod_{i=1}^{N} F_{j, N}(\xi)=0$ & (14b) \\
\hline 2 & $\mathfrak{S}_{0}^{\circ}$ & $\left(2-2 \cos \xi-\omega^{2}\right) \prod_{j=1}^{N} F_{j, N}(\xi)=0$ & (24) \\
\hline 3 & $\mathfrak{s}_{0}^{:}$ & $\prod_{j=1}^{N} F_{j, N}(\xi)-\prod_{j=1}^{N-1} F_{j, N-1}(\xi)=0$ & (29) \\
\hline 4 & $\Xi_{\odot}$ & $\prod_{j=1}^{N} F_{j, N}(\xi)-4 \prod_{j=1}^{N-2} F_{j, N-2}(\xi)-2^{N+1}=0$ & (30) \\
\hline 1 & $\mathfrak{I}^{\bullet}$ & $\prod_{j=1}^{N} F_{j, N}(\xi)=0$ & (38) \\
\hline 2 & $\mathfrak{I}_{0}^{\circ}$ & $\prod_{j=1}^{N} F_{j, N}(\xi)+4 \prod_{j=1}^{N-2} F_{j, N-2}(\xi)-4 \prod_{j=1}^{N-1} F_{j, N-1}(\xi)=0$ & (41) \\
\hline 3 & $\mathfrak{I}_{0}^{0}$ & $\prod_{j=1}^{N} F_{j, N}(\xi)-2 \prod_{j=1}^{N-1} F_{j, N-1}(\xi)=0$ & (54) \\
\hline 4 & $\mathfrak{I} \odot$ & $\prod_{j=1}^{N} F_{j, N}(\xi)-(2 \cos \xi)^{2} \prod_{j=1}^{N-2} F_{j, N-2}(\xi)-2(2 \cos \xi)^{N}=0$ & (30) \\
\hline 1 & $\hat{\Theta}_{\bullet}^{\bullet}$ & $\prod_{j=1}^{N} F_{j, N}(\xi)=0$ & (66c) \\
\hline 2 & $\hat{\Theta}_{\circ}^{\circ}$ & $\prod_{j=1}^{N} F_{j, N}(\xi)+\Gamma_{0}^{2} \prod_{j=1}^{N-2} F_{j, N-2}(\xi)-2 \Gamma_{0} \prod_{j=1}^{N-1} F_{j, N-1}(\xi)=0$ & (69) \\
\hline 3 & $\hat{\mathfrak{\epsilon}}_{0}^{\bullet}$ & $\prod_{j=1}^{N} F_{j, N}(\xi)-\Gamma_{0} \prod_{j=1}^{N-1} F_{j, N-1}(\xi)=0$ & (85) \\
\hline 4 & $\hat{\Xi}_{\odot}$ & $\prod_{j=1}^{N} F_{j, N}(\xi)-\Gamma(\xi)^{2} \prod_{j=1}^{N-2} F_{j, N-2}(\xi)-2 \Gamma(\xi)^{N}=0$ & (30) \\
\hline
\end{tabular}

Several applications of the expressions catalogued in this paper appear in a series of forthcoming papers by the author on dislocation kinetics in lattice strips, mode III brittle fracture of lattice strips (for example, [24]), wave propagation in bifurcated lattice waveguides $[25,26]$ and scattering by an infinite array of parallel semi-infinite cracks and rigid constraints. The results analogous to those presented in this paper for the honeycomb lattice structure appear in [85].

\section{Acknowledgements}

The research work was partially supported by IITK/ME/ 20080334 and IITK/ME/20090027. The author thanks an anonymous reviewer for the constructive comments and suggestions.

\section{Appendix 1: Chebyshev polynomials}

Let $n$ be a positive integer. Recall that [56-58]

$$
\begin{aligned}
T_{n}(\vartheta) & =\frac{1}{2}\left(\left(\vartheta+\sqrt{\vartheta^{2}-1}\right)^{n}+\left(\vartheta-\sqrt{\vartheta^{2}-1}\right)^{n}\right), U_{n}(\vartheta) \\
& =\frac{1}{2}\left(\left(\vartheta+\sqrt{\vartheta^{2}-1}\right)^{n}-\left(\vartheta-\sqrt{\vartheta^{2}-1}\right)^{n}\right) / \sqrt{\vartheta^{2}-1}, \\
V_{n}(\vartheta) & =\left(w^{n+1}+w^{-n}\right) /(w+1), W_{n}(\vartheta) \\
& =\left(w^{n+1}-w^{-n}\right) /(w-1), \vartheta=\frac{1}{2}\left(w+w^{-1}\right) .
\end{aligned}
$$

The following identities are useful also:

$$
\begin{aligned}
& U_{n}=2 \vartheta U_{n-1}-U_{n-2}, 2 T_{n}=V_{n}+V_{n-1}, V_{n}=U_{n}-U_{n-1}, \\
& W_{n}=U_{n}+U_{n-1}, \\
& 2 T_{n}=U_{n}-U_{n-2}, U_{n}^{2}=1+U_{n-1} U_{n+1}, U_{n}( \pm 1)=(n+1)(-1)^{\left(\frac{1}{2}-\frac{1}{2}\right) n} .
\end{aligned}
$$

A few more identities can be proved [58] as follows:

$$
V_{n} W_{n}=\frac{\cos \left(n+\frac{1}{2}\right) \eta}{\cos \frac{1}{2} \eta} \frac{\sin \left(n+\frac{1}{2}\right) \eta}{\sin \frac{1}{2} \eta}=\frac{\sin (2 n+1) \eta}{\sin \eta}=U_{2 n}
$$


$2 T_{n+1} U_{n}=2 \cos (n+1) \eta \frac{\sin (n+1) \eta}{\sin \eta}=\frac{\sin (2 n+2) \eta}{\sin \eta}=U_{2 n+1}$,

$$
\begin{aligned}
V_{n-1} W_{n}+V_{n} W_{n-1} & =\frac{\cos \left(n-\frac{1}{2}\right) \eta}{\cos \frac{1}{2} \eta} \frac{\sin \left(n+\frac{1}{2}\right) \eta}{\sin \frac{1}{2} \eta} \\
& +\frac{\sin \left(n-\frac{1}{2}\right) \eta}{\sin \frac{1}{2} \eta} \frac{\cos \left(n+\frac{1}{2}\right) \eta}{\cos \frac{1}{2} \eta}=U_{2 n-1}
\end{aligned}
$$

$$
\begin{aligned}
T_{n} U_{n}+T_{n+1} U_{n-1} & =\cos n \eta \frac{\sin (n+1) \eta}{\sin \eta} \\
& +\cos (n+1) \eta \frac{\sin n \eta}{\sin \eta}=U_{2 n}
\end{aligned}
$$$$
U_{n-1}^{2}=\frac{\sin ^{2} n \eta}{\sin ^{2} \eta}=\frac{1}{2} \frac{1-\cos 2 n \eta}{1-\cos ^{2} \eta}=-\frac{1}{2} \frac{1-T_{2 n}}{\left(\vartheta^{2}-1\right)},
$$

$$
W_{n}^{2}=\frac{\sin ^{2}\left(n+\frac{1}{2}\right) \eta}{\sin ^{2} \frac{1}{2} \eta}=\frac{1-\cos (2 n+1) \eta}{1-\cos \eta}=-\frac{1-T_{2 n+1}}{(\vartheta-1)} .
$$

\section{Appendix 2: Auxiliary identities and some derivations}

Using the elementary properties of trigonometric functions and geometric series,

$\sum_{j=1}^{N} \sin j \eta=-i \frac{1}{2}\left(\sum_{j=1}^{N} e^{i j \eta}-\sum_{j=1}^{N} e^{-i j \eta}\right)=\frac{\sin (N+1) \frac{1}{2} \eta \sin N \frac{1}{2} \eta}{\sin \frac{1}{2} \eta}$

$$
\text { and } \begin{aligned}
\sum_{j=1}^{N} \cos j \eta & =\frac{1}{2}\left(\sum_{j=1}^{N} e^{i j \eta}+\sum_{j=1}^{N} e^{-i j \eta}\right) \\
& =\frac{\cos (N+1) \frac{1}{2} \eta \sin N \frac{1}{2} \eta}{\sin \frac{1}{2} \eta}
\end{aligned}
$$

so that

$$
\sum_{j=1}^{N} \sin ^{2} j \eta=\frac{1}{2} \sum_{j=1}^{N}(1-\cos 2 j \eta)=\frac{1}{2} N-\frac{1}{2} \frac{\cos (N+1) \eta \sin N \eta}{\sin \eta}
$$

$\sum_{j=1}^{N} \cos ^{2} j \eta=\frac{1}{2} \sum_{j=1}^{N}(1+\cos 2 j \eta)=\frac{1}{2} N+\frac{1}{2} \frac{\cos (N+1) \eta \sin N \eta}{\sin \eta}$

$$
\begin{aligned}
\sum_{j=1}^{N} \cos ^{2}\left(j-\frac{1}{2}\right) \eta & =\frac{1}{2} \sum_{j=1}^{N}(1+\cos (2 j-1) \eta) \\
& =\frac{1}{2} N+\frac{1}{2} \frac{\cos N \eta \sin N \eta}{\sin \eta}
\end{aligned}
$$

In terms of Chebyshev polynomials [56-58], with $\cos \eta=$ $\vartheta$ as the argument (suppressed), using $(102 \mathrm{~b})_{1}$,

$$
\begin{aligned}
& \sum_{j=1}^{N} \sin ^{2} j \eta=\frac{1}{2} N-\frac{1}{2} T_{N+1} U_{N-1}=\frac{1}{2} N-\frac{1}{4}\left(U_{N+1}-U_{N-1}\right) U_{N-1}, \\
& \sum_{j=1}^{N} \cos ^{2} j \eta==\frac{1}{2} N+\frac{1}{2} T_{N+1} U_{N-1}=\frac{1}{2} N+\frac{1}{4}\left(U_{N+1}-U_{N-1}\right) U_{N-1},
\end{aligned}
$$

$$
\sum_{j=1}^{N} \cos ^{2}\left(j-\frac{1}{2}\right) \eta=\frac{1}{2} N+\frac{1}{4}\left(U_{N}-U_{N-2}\right) U_{N-1} .
$$

Also,

$$
\begin{aligned}
\sum_{v=1}^{N} \sin j \eta \sin (j-1) \eta & =\cos \eta \sum_{v=1}^{N} \sin ^{2} j \eta-\frac{1}{2} \sin \eta \sum_{v=1}^{N} \sin 2 j \eta \\
& =\frac{1}{2} N \cos \eta-\frac{1}{4} \cos \eta\left(U_{N+1}-U_{N-1}\right) U_{N-1} \\
& -\frac{1}{2} \sin ^{2} \eta U_{N} U_{N-1}(\text { by }(106 \mathrm{a}),(104 \mathrm{a})) \\
& =\frac{1}{2}\left(N \cos \eta-\frac{1}{2} U_{N-1}\left(U_{N}-U_{N-2}\right)\right)\left(\mathrm{by}(102 \mathrm{a})_{1}\right) .
\end{aligned}
$$

In the following also, the argument $\vartheta$ (21) (with (18), (44), and (68)) of the Chebyshev polynomials is suppressed, and $\eta$ is used in place of $\eta_{\kappa}$, for brevity.

\section{Appendix 2.1: $\mathfrak{\Im}_{N}$}

By (106a), the normalization constant in (14b) can be obtained as

$$
\sum_{v \in \mathbb{Z}_{1}^{N}} \sin ^{2} v \eta=\frac{1}{2} N+\frac{1}{2} U_{N-1}^{2}\left(\text { by }(102 \mathrm{a})_{1} \text { and }(15)\right),
$$

followed by (102b) 2 and (15). By (106c), the normalization constant in (24) follows as

$$
\begin{aligned}
\sum_{v \in \mathbb{Z}_{1}^{N}} \cos ^{2}\left(v-\frac{1}{2}\right) \eta= & \frac{1}{2} N(\text { by }(25) \text { for } \vartheta \neq 1, \\
& \text { with } \left.(102 \mathrm{~b})_{3} \text { for } \vartheta=1\right) .
\end{aligned}
$$

By (106c), the normalization constant in (29) follows as

$$
\begin{aligned}
\sum_{v \in \mathbb{Z}_{1}^{N}} \cos ^{2}\left(v-\frac{1}{2}\right) \eta= & \frac{1}{2} N+\frac{1}{4}\left(U_{N}^{2}+1-U_{N-1}^{2}\right) \\
& \left(\text { by }(28) \text { and }(102 \mathrm{~b})_{3}\right),
\end{aligned}
$$

leading to (29) after applying (28). 


\section{Appendix 2.2: $\mathfrak{I}_{N}$}

The normalization constant in (38) follows from (107). Using (106a), (106b) and (106d), the normalization constant in (41) is given by

$$
\begin{aligned}
C_{N}^{-2}= & \cos ^{2} \xi \sum_{v=1}^{N} \sin ^{2} v \eta+\sum_{v=1}^{N} \sin ^{2}(v-1) \eta \\
& -2 \cos \xi \sum_{v=1}^{N} \sin v \eta \sin (v-1) \eta \\
= & \frac{1}{2} N\left(1+\cos ^{2} \xi-2 \vartheta \cos \xi\right)-\frac{1}{2}+S, \text { where } \\
S & =-\frac{1}{4} \cos ^{2} \xi\left(U_{N+1}-U_{N-1}\right) U_{N-1}-\frac{1}{4}\left(U_{N}-U_{N-2}\right) U_{N-2} \\
+ & \frac{1}{2} \cos \xi U_{N-1}\left(U_{N}-U_{N-2}\right) .
\end{aligned}
$$

The expression of $S$ can be simplified as

$$
\begin{aligned}
S= & -\frac{1}{4} \cos ^{2} \xi\left(\left(U_{N+1}-U_{N-1}\right) U_{N-1}-U_{N}\left(U_{N}-U_{N-2}\right)\right)(\text { by }(39)) \\
= & -\frac{1}{4} \cos ^{2} \xi\left(\left(U_{N+1}-U_{N-1}\right) U_{N-1}\right. \\
& \left.-\left(1+U_{N-1} U_{N+1}-U_{N} U_{N-2}\right)\right)\left(\text { by }(102 \mathrm{~b})_{3}\right) \\
= & \frac{1}{2} \cos ^{2} \xi\left(\text { again by }(102 \mathrm{~b})_{3}\right) .
\end{aligned}
$$

The normalization constant in (45) is derived as follows. By (43) and (102a) ${ }_{1}$,

$$
(1+\cos \xi) U_{N}+(-\mathscr{Q}-1+\cos \xi) U_{N-1}=0 .
$$

Employing (106c),

$$
\begin{aligned}
C_{N}^{-2}= & 2 \sum_{v=1}^{N} \cos ^{2}\left(v-\frac{1}{2}\right) \eta=N+\frac{1}{2}(1-\cos \xi) \\
& \left(U_{N}+U_{N-1}\right) U_{N-1}(\text { by }(43)) \\
= & N+\frac{1}{2} \frac{1-\cos \xi}{1+\cos \xi}(2+2) U_{N-1}^{2}(\text { by (111a) }) .
\end{aligned}
$$

Using (106a) and (46), the normalization constant in (47) can be expressed as

$$
\begin{aligned}
C_{N}^{-2} & =2 \sum_{v=1}^{N} \sin ^{2} v \eta=N-\frac{1}{2}\left(U_{N+1}-U_{N-1}\right) \cos \xi \\
U_{N} & =N-\frac{1}{2} \cos \xi(\mathscr{Q}-2 \cos \xi) U_{N}^{2}\left(\operatorname{by}(102 \mathrm{a})_{1}\right) \\
& =N-\frac{1}{2}\left(2 \cos \xi-\cos ^{2} \xi-1+\sin ^{2} \xi\right) U_{N}^{2} .
\end{aligned}
$$

By (102a) $)_{1}$, using (46), $U_{N+1}=(\mathscr{2}-\cos \xi) U_{N}$; also by (102b) $)_{3}$, using (46), $U_{N}^{2}=1+(\mathscr{Q}-\cos \xi) \cos \xi U_{N}^{2}$, so that $\left(1-2 \cos \xi+\cos ^{2} \xi\right) U_{N}^{2}=1$. Hence, the normalization in
(47) follows. The normalization constant in (49) is considered in the same manner. By (48) and (102a) $)_{1}$,

$$
(\cos \xi-1) U_{N}+(\mathscr{Q}-1-\cos \xi) U_{N-1}=0,
$$

so that (106c) leads to

$$
\begin{aligned}
C_{N}^{-2} & =N+\frac{1}{2}\left(U_{N}-U_{N-1}+U_{N-1}-U_{N-2}\right) U_{N-1} \\
& =N+\frac{1}{2} \frac{1+\cos \xi}{1-\cos \xi}(\mathscr{Q}-2) U_{N-1}^{2}(\text { by }(48) \text { and }(113 \mathrm{a})) .
\end{aligned}
$$

The normalization constant in (51) is derived below. Using $(102 a)_{1}$ in (50),

$$
(\mathscr{2} \cos \xi-2) U_{N}+(\mathscr{Q}-2 \cos \xi) U_{N-1}=0,
$$

while using (106b) and (50),

$$
\begin{aligned}
C_{N}^{-2} & =1+2 \sum_{v=1}^{N} \cos ^{2} v \eta=1+N+\frac{1}{2}\left(U_{N+1}-U_{N-1}\right) U_{N-1} \\
& =1+N+\frac{1}{2}\left(U_{N}^{2}-1-U_{N-1}^{2}\right)\left(\mathrm{by}(102 \mathrm{~b})_{3}\right) \\
& =N+\frac{1}{2}+\frac{1}{2} \frac{(1-\cos \xi)(1+\cos \xi)}{(2 \cos \xi-2)^{2}}(2+2) \\
& (\mathscr{Q}-2) U_{N-1}^{2}(\text { by }(114 \mathrm{a}))
\end{aligned}
$$

resulting in (51). The derivation of the normalization constant in (54) is almost identical to that presented for (47) (see (112)).

\section{Appendix 2.3: $\hat{\mathfrak{\Xi}}_{N}$}

The normalization constant in (66c) follows from (107) as in the case of the corresponding triangular lattice waveguide. Following (110a), using (106a), (106b) and (106d), the normalization constant in (69) is given by

$$
\begin{aligned}
C_{N}^{-2}= & \frac{1}{2} N\left(\Gamma(\xi)^{2}+\Gamma_{0}^{2}-2 \vartheta \Gamma_{0} \Gamma(\xi)\right)-\frac{1}{2} \Gamma_{0}+S, \text { where } \\
S= & -\frac{1}{4} \Gamma(\xi)^{2}\left(U_{N+1}-U_{N-1}\right) U_{N-1} \\
& \quad-\frac{1}{4} \Gamma_{0}^{2}\left(U_{N}-U_{N-2}\right) U_{N-2}+\frac{1}{2} \Gamma_{0} \Gamma(\xi) U_{N-1}\left(U_{N}-U_{N-2}\right) .
\end{aligned}
$$

The expression of $S$ can be simplified as in the same way as (110b) to yield $S=\frac{1}{2} \Gamma(\xi)^{2}$. The normalization constant in (73) is derived as follows. By (71) and (102a),

$$
(2+2 \gamma(1+\cos \xi)) U_{N}+\left(\Gamma(\xi)-\Gamma_{0}(\mathscr{2}+1)\right) U_{N-1}=0 .
$$


Following (111b), employing (106c),

$$
C_{N}^{-2}=N+\frac{1}{2} \Gamma_{0}^{-1}\left(\Gamma_{0}-\Gamma(\xi)\right)\left(U_{N}+U_{N-1}\right) U_{N-1}(\text { by }(71)),
$$

which leads to (73) using (116a). Following (112), using (106a) and (74), the normalization constant in (75) can be expressed as

$$
C_{N}^{-2}=N-\frac{1}{2} \frac{\Gamma(\xi)}{\Gamma_{0}}\left(\mathscr{Q}-2 \frac{\Gamma(\xi)}{\Gamma_{0}}\right) U_{N}^{2}\left(\text { by }(102 \mathrm{a})_{1}\right) .
$$

By (102a) 1 , using (74), $U_{N+1}=(\mathscr{Q}-\alpha) U_{N}, \alpha=\Gamma(\xi) / \Gamma_{0}$; also by $(102 \mathrm{~b})_{3}$, using (74), $U_{N}^{2}=1+U_{N+1} U_{N-1}=1+$ $(\mathscr{2}-\alpha) \alpha U_{N}^{2}$, so that $\left(1-\mathscr{2} \alpha+\alpha^{2}\right) U_{N}^{2}=1$. Hence, (76) follows.

Following (113b), by (77) and (102a) $)_{1}$,

$$
2 \gamma(1-\cos \xi) U_{N}+\left(\Gamma(\xi)-\Gamma_{0}(\mathscr{Q}-1)\right) U_{N-1}=0,
$$

so that (106c) leads to

$$
\begin{aligned}
C_{N}^{-2} & =N+\frac{1}{2} \Gamma_{0}^{-1}\left(\Gamma_{0}+\Gamma(\xi)\right)\left(U_{N}-U_{N-1}\right) U_{N-1}(\text { by }(71)) \\
& =N+\frac{1}{2} \frac{2+2 \gamma(1+\cos \xi)}{2 \gamma(1-\cos \xi)}(2-2) U_{N-1}^{2}(\text { by }(118 \mathrm{a}))
\end{aligned}
$$

yielding (78). The normalization constant in (80) is derived here. Using (102a) $)_{1}$ in (79),

$$
\left(2 \Gamma(\xi)-2 \Gamma_{0}\right) U_{N}+\left(\mathscr{2} \Gamma_{0}-2 \Gamma(\xi)\right) U_{N-1}=0,
$$

while following (114b), using (106b) and (79),

$$
\begin{aligned}
C_{N}^{-2} & =1+N+\frac{1}{2}\left(U_{N}^{2}-1-U_{N-1}^{2}\right)\left(\text { by }(102 \mathrm{~b})_{3}\right) \\
& =N+\frac{1}{2}+2 \gamma \frac{\left(1-\cos \xi+\gamma \sin ^{2} \xi\right)}{(\mathscr{Q}-2+2 \gamma(2 \cos \xi-2))^{2}} \\
& (\mathscr{Q}+2)(\mathscr{Q}-2) U_{N-1}^{2}(\text { by }(119 \mathrm{a})),
\end{aligned}
$$

resulting in (81). Similar to the triangular lattice, the normalization constant in (85) can be derived in almost an identical manner as that presented for (75) (see (117)).

\section{References}

[1] Kolsky H 1964 Stress waves in solids. J. Sound Vibr. 1(1): 88-110, doi:10.1016/0022-460X(64)90008-2

[2] Miklowitz J 1978 The theory of elastic waves and waveguides. Applied mathematics and mechanics. New York: North Holland

[3] Giebe E and Blechschmidt E 1933 Experimentelle und theoretische Untersuchungen uber Dehnungseigenschwingungen von Stben und Rohren. II. Ann. Phys. 410(5): 457-485, doi:10. 1002/andp.19334100502

[4] Rohrich K 1932 Ausbreitungsgeschwindigkeit ultraakustischer Schwingungen in zylindrischen Stben. Z. Phys. 73(11-12): 813-832, doi:10.1007/BF01344227

[5] Schoeneck H 1934 Experimentelle Untersuchungen der Schwingungen zylindrischer Einzelkristalle bei hohen elastischen Frequenzen. Z. Phys. 92(5-6): 390-406, doi:10. 1007/BF01340823

[6] Chree C 1886 Longitudinal vibrations of a circular bar. $Q$. J. Pure Appl. Math. 21: 287-298

[7] Pochhammer L 1876 Ueber die Fortpflanzungsgeschwindigkeiten kleiner Schwingungen in einem unbegrenzten isotropen Kreiscylinder. J. di Reine Angew. Math. 81: $324-336$

[8] Rayleigh L 1885 On waves propagated along the plane surface of an elastic solid. Proc. London Math. Soc. s117(1): 4-11, doi:10.1112/plms/s1-17.1.4

[9] Rayleigh L 1888 On the free vibrations of an infinite plate of homogeneous isotropic elastic matter. Proc. London Math. Soc. S120(1): 225-237, doi:10.1112/plms/s1-20.1.225

[10] Lamb H 1903 On the propagation of tremors over the surface of an elastic solid. [Abstract]. R. Soc. London Proc. Ser. I 72: 128-130

[11] Lamb H 1917 On waves in an elastic plate. R. Soc. London Proc. Ser. A 93: 114-128, doi:10.1098/rspa.1917.0008

[12] Brillouin L 1953 Wave propagation in periodic structures: electric filters and crystal lattices. New York: Dover

[13] Bancroft D 1941 The velocity of longitudinal waves in cylindrical bars. Phys. Rev. 59(7): 588-593, doi:10.1103/ PhysRev.59.588

[14] Love A E H 1927 A treatise on the mathematical theory of elasticity. 4th ed. Mineola: Dover

[15] Davies R M 1948 A critical study of the Hopkinson pressure bar. Philos. Trans. R. S. London A: Math. Phys. Eng. Sci. 240(821): 375-457, doi:10.1098/rsta.1948.0001

[16] Hudson G E 1943 Dispersion of elastic waves in solid circular cylinders. Phys. Rev. 63(1-2): 46-51, doi:10.1103/ PhysRev.63.46

[17] Onoe M, McNiven H D and Mindlin R D 1962 Dispersion of axially symmetric waves in elastic rods. Trans. ASME: J. Appl. Mech. 29: 729, doi:10.1115/1.3640661

[18] Collin R 1991 Field theory of guided waves. IEEE/OUP series on electromagnetic wave theory. New York: IEEE Press

[19] Marcuvitz N 1951 Waveguide handbook. IEE electromagnetic waves series. London: Institution of Electrical Engineers and P Peregrinus

[20] Degnan J J 1976 The waveguide laser: a review. Appl. Phys. 11(1): 1-33, doi:10.1007/BF00895012

[21] Karbowiak A 1959 Radiation and guided waves. IEEE Trans. Antennas Propag. 7: 191-200, doi:10.1109/TAP.1959.1144748

[22] Young M 1992 Optics and lasers: including fibers and optical waveguides. Optik, Laser, Wellenleiter: Mit zahlreichen Beispielen und 133 Aufgaben mit vollständigen Lösungen. New York: Springer

[23] Mittra R and Lee S 1971 Analytical techniques in the theory of guided waves. Macmillan series in electrical science. New York: Macmillan

[24] Sharma B L 2016 On energy balance and the structure of radiated waves in kinetics of crystalline defects. J. Mech. Phys. Solids 96: 88-120, doi:10.1016/j.jmps.2016.05.036 
[25] Sharma B L 2016 Transmission of anti-plane shear waves in bifurcated waveguides on close packed planes, pp. 1-28 (under review)

[26] Sharma B L 2016e Wave propagation in bifurcated waveguides of square lattice strips. SIAM J. Appl. Math. 76(4): 1355-1381, doi:10.1137/15M1051464

[27] Williams W 1957 Step discontinuities in waveguides. IRE Trans. Antennas Propag. 5(2): 191-198, doi:10.1109/TAP. 1957.1144499

[28] Born M and von Karman T 1912 On fluctuations in spatial grids. Phys. Z. 13: 297-309

[29] Born M and Huang K 1985 Dynamical theory of crystal lattices. Oxford Classic Texts in the Physical Sciences. New York: The Clarendon Press, Oxford University Press

[30] Lifshitz I M 1956 Some problems of the dynamic theory of non-ideal crystal lattices. Il Nuovo Cimento Suppl. 3: 716-733

[31] Lifshitz I M and Kosevich A M 1966 The dynamics of a crystal lattice with defects. Rep. Prog. Phys. 29: 217-254

[32] Yu X and Leitner D M 2003 Anomalous difusion of vibrational energy in proteins. J. Chem. Phys. 119(23): 12673-12679, doi:10.1063/1.1626636

[33] Sándor E 1962 Lattice vibrations of molecular chains. Acta Crystallogr. 15(5): 463-473, doi:10.1107/ S0365110X62001164

[34] Deymier P and Dobrzynski L 2013 Discrete one-dimensional phononic and resonant crystals. In: Deymier P A (Ed.) Acoustic metamaterials and phononic crystals, vol. 173, Springer Series in Solid-State Sciences, Berlin Heidelberg: Springer, pp. 13-44, doi:10.1007/978-3-642-31232-8_2

[35] Pennec Y et al 2010 Simultaneous existence of phononic and photonic band gaps in periodic crystal slabs. Opt. Express 18(13): 14301-14310, doi:10.1364/OE.18.014301

[36] Galli M et al 2004 Excitation of radiative and evanescent defect modes in linear photonic crystal waveguides. Phys. Rev. B 70(8): 081307, doi:10.1103/PhysRevB.70.081307

[37] Kosevich Y A 2008 Multichannel propagation and scattering of phonons and photons in low-dimension nanostructures. Physc-Usp 51(8): 848

[38] Mardaani M and Esfarjani K 2005 Analytical results on ballistic transport in a periodic molecular wire. Chem. Phys. 317(1): 43-48, doi:10.1016/j.chemphys.2005.05.031

[39] Narayan B 2016 End modes in arrays of modulated SuSchrieffer-Heeger chains. Pramana 87(2): 1-7, doi:10.1007/ s12043-016-1217-7

[40] Sørensen H H B et al 2009 Efficient wave-function matching approach for quantum transport calculations. Phys. Rev. B 79(20): 205322, doi:10.1103/PhysRevB.79.205322

[41] Zwierzycki M et al 2008 Calculating scattering matrices by wave function matching. Phys. Status Solidi (b) 245(4): 623-640, doi:10.1002/pssb.200743359

[42] Bourahla B, Nafa O and Tigrine R 2011 Phonon scattering in quasi-one-dimensional structure. Phys. B: Condens. Matter. 406(4): 725-730, doi:10.1016/j.physb.2010.11.006

[43] Cahill D G et al 2003 Nanoscale thermal transport. J. Appl. Phys. 93(2): 793-818, doi:10.1063/1.1524305

[44] Kosevich Y A, Feher A and Syrkin E S 2008 Resonance absorption, reflection, transmission of phonons and heat transfer through interface between two solids. Low Temp. Phys. 34(7): 575-582, doi:10.1063/1.2957011
[45] Li D et al 2003 Thermal conductivity of individual silicon nanowires. Appl. Phys. Lett. 83(14): 2934-2936, doi:10. 1063/1.1616981

[46] Belhadi M et al 2000 The scattering and transmission of elastic waves in quasi-two-dimensional planar waveguides with linear defect boundaries. Eur. Phys. J. B-Condens. Matter Complex Syst. 15(3): 435-443, doi:10.1007/s100510051144

[47] Fellay A et al 1997 Scattering of vibrational waves in perturbed quasi-one-dimensional multichannel waveguides. Phys. Rev. B 55(3): 1707-1717, doi:10.1103/PhysRevB.55.1707

[48] Vasseur J O et al 1998 Experimental evidence for the existence of absolute acoustic band gaps in twodimensional periodic composite media. J. Phys. Condens. Matter 10(27): 6051

[49] Ashokan V et al 2014 Ballistic transport of spin waves incident from cobalt leads across cobalt-gadolinium alloy nanojunctions. J. Magn. Magn. Mater. 363: 66-76, doi:10. 1016/j.jmmm.2014.03.064

[50] Gopalan S, Rice T M and Sigrist M 1994 Spin ladders with spin gaps: a description of a class of cuprates. Phys. Rev. B 49(13): 8901-8910, doi:10.1103/PhysRevB.49.8901

[51] Rozhkov A et al 2011 Electronic properties of mesoscopic graphene structures: charge confinement and control of spin and charge transport. Phys. Rep. 503(2-3): 77-114, doi:10. 1016/j.physrep.2011.02.002

[52] Dean P 1963 The vibrations of three two-dimensional lattices. Proc. Camb. Philos. Soc. 59: 383-396

[53] Grünbaum B and Shephard G C 1989 Tilings and patterns: an introduction. Sections 2.1 and 2.2. New York: W. H. Freeman and Company

[54] Maradudin A A et al 1971 Theory of lattice dynamics in the harmonic approximation, 2nd. ed. New York: Academic Press

[55] Slepyan L I 2002 Models and phenomena in fracture mechanics. New York, Berlin, Heidelberg: Springer

[56] Chebyshev P L 1854 Théorie des mécanismes connus sous le nom de parallélogrammes. Mém. Acad. Sci. Pétersb 7: 539-568

[57] Fox L and Parker I B 1968 Chebyshev polynomials in numerical analysis. London-New York-Toronto: Oxford University Press

[58] Mason J C and Handscomb D C 2003 Chebyshev polynomials. Boca Raton, FL: Chapman \& Hall/CRC

[59] Bavinck H and Dieterman H 2000 Closed-form dynamic response of damped mass-spring cascades. J. Comput. Appl. Math. 114(2): 291-303, doi:10.1016/S0377-0427(99)00206$\mathrm{X}$

[60] Dow M 2003 Explicit inverses of Toeplitz and associated matrices. ANZIAM J. 44: E185-E215

[61] Yueh W-C and Cheng S S 2008 Explicit eigenvalues and inverses of tridiagonal Toeplitz matrices with four perturbed corners. ANZIAM J. 49(3): 361-387, doi:10.1017/S1446181 108000102

[62] Bavinck H 1995 On the zeros of certain linear combinations of Chebyshev polynomials. J. Comput. Appl. Math. 65(1-3): 19-26, doi:10.1016/0377-0427(95)00098-4

[63] Kouachi S 2006 Eigenvalues and eigenvectors of tridiagonal matrices. Electron. J. Lin. Algebra 15: 115-133

[64] Sharma B L 2015 Diffraction of waves on square lattice by semi-infinite crack. SIAM J. Appl. Math. 75(3): 1171-1192, doi:10.1137/140985093 
[65] Sharma B L 2016 Diffraction of waves on triangular lattice by a semi-infinite rigid constraint and crack. Int. J. Solids Struct. 80: 465-485, doi:10.1016/j.ijsolstr.2015.10.008

[66] Sharma B L 2015 Discrete Sommerfeld diffraction problems on hexagonal lattice with a zigzag semi-infinite crack and rigid constraint. Z. Angew. Math. Phys. 66(6): 3591-3625, doi:10.1007/s00033-015-0574-2

[67] Meek D 1980 The inverses of some matrices deviating slightly from a symmetric, tridiagonal, Toeplitz form. SIAM J. Num. Anal. 17(1): 39-43, doi:10.1137/0717006

[68] Shin B C 1997 A formula for eigenpairs of certain symmetric tridiagonal matrices. Bull. Aust. Math. Soc. 55(2): 249-254, doi:10.1017/S0004972700033918

[69] Willms A R 2008 Analytic results for the eigenvalues of certain tridiagonal matrices. SIAM J. Matrix Anal. Appl. 30(2): 639-656, doi:10.1137/070695411

[70] Macrae A 1964 Surface atom vibrations. Surf. Sci. 2: 522-526, doi:10.1016/0039-6028(64)90095-0

[71] Maradudin A A and Melngailis J 1964 Some dynamical properties of surface atoms. Phys. Rev. 133(4A): A1188A1193, doi:10.1103/PhysRev.133.A1188

[72] Wallis R 1964 Surface effects on lattice vibrations. Surf. Sci. 2: 146-155, doi:10.1016/0039-6028(64)90053-6

[73] Wallis R 1974 Lattice dynamics of crystal surfaces. Prog. Surf. Sci. 4: 233-367, doi:10.1016/S0079-6816(74)80007-9

[74] Duparc O H, Sanz-Velasco E and Velasco V R 1984 Elastic surface waves in crystals with overlayers: cubic symmetry. Phys Rev. B 30(4): 2042-2048, doi:10.1103/PhysRevB.30. 2042
[75] Tiersten H F 1969 Elastic surface waves guided by thin films. J. Appl. Phys. 40(2): 770-789, doi:10.1063/1.1657463

[76] Wu B et al 2015 Surface effects on anti-plane shear waves propagating in magneto-electro-elastic nanoplates. Smart Mater. Struct. 24(9): 095017

[77] Kantorovich L and Krylov V 1958 Approximate methods of higher analysis. Translated by Curtis D. Benster. New York: Interscience

[78] Achenbach J D 1973 Wave propagation in elastic solids. Amsterdam: North-Holland

[79] Bilbao S 2004 Wave and scattering methods for numerical simulation. Hoboken: Wiley

[80] Collatz L 1960 The numerical treatment of differential equations, 3rd ed. Berlin: Springer

[81] Horiguchi T 1972 Lattice Green's functions for the triangular and honeycomb lattices. J. Math. Phys. 13: 1411-1419

[82] Levy H and Lessman F 1992 Finite difference equations. New York: Dover

[83] Babuska I, Vitasek E and Kroupa F 1960a Some applications of the discrete Fourier transform to problems of crystal lattice deformation I. Czech. J. Phys. B 10: 419-427

[84] Babuska I, Vitasek E and Kroupa F 1960b Some applications of the discrete Fourier transform to problems of crystal lattice deformation II. Czech J. Phys. B 10: 488-504

[85] Sharma B L 2016 On linear waveguides of zigzag honeycomb lattice. In: Waves in random and complex media, pp. 1-39 (under review) 